COMMUNICATIONS IN

ANALYSIS AND GEOMETRY

Volume 13, Number 2, 253-285, 2005

\title{
Canonical metrics on stable vector bundles
}

\author{
XIAOWEI WANG
}

This paper is the sequel to [W], we prove that balanced metrics obtained in $[\mathrm{W}]$ on a Mumford stable vector bundle over a projective manifold converge to a solution of the weakly Hermitian-Einstein equation.

\section{Introduction.}

The problem of constructing moduli space of vector bundles over a projective manifold has attracted many mathematicians for decades. In mid 60's Mumford first constructed the moduli space of vector bundles over algebraic curves via his celebrated GIT machinery. Later, in early 80's Atiyah and Bott found an infinite dimensional symplectic quotient description of this moduli space. Since then, we have learned quiet a lot from the work of Kirwan, Guillemin and Sternberg in 80's that finite dimensional GIT quotient is equivalent to symplectic quotient. A question that is remaining is how Atiyah-Bott's infinite dimensional symplectic quotient is approximated by Mumford's finite dimensional GIT quotient. This is the question we are studying in this paper.

To state our main result, let $\left(X, \mathcal{O}_{X}(1)\right)$ be a projective manifold polarized by an ample line bundle $\mathcal{O}_{X}(1)$ and $\mathcal{E}$ be an irreducible holomorphic vector bundle of rank $r$ on $X$. Then by Kodaira embedding theorem, we know that for $k$ sufficient large a basis $\left\{S_{\alpha}\right\}$ of $\operatorname{dim} H^{0}\left(X, \mathcal{E}(k):=\mathcal{E} \otimes \mathcal{O}_{X}(k)\right)$ will give rise to an embedding

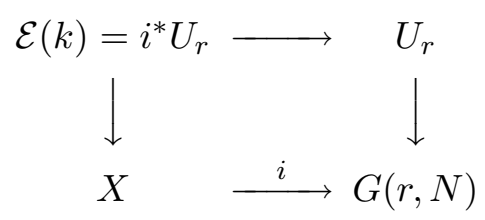

where $N:=\operatorname{dim} H^{0}(X, \mathcal{E}(k))$ and $U_{r}$ is the dual of universal subbundle. In $[\mathrm{W}]$, we have shown

Theorem 1.1. $\mathcal{E}$ is Gieseker stable if and only if there is a number $k_{0}$ such that for $k>k_{0}$, the $k$ th-embedding given as above can be moved to 
a balanced place, i.e. there is a $g \in S L(N)$ which is unique up to left translation by $S U(N)$ such that:

$$
\frac{1}{V} \int_{g \cdot X} z\left(z^{*} z\right)^{-1} z^{*} d V=\frac{r}{N} I_{N \times N}
$$

where $z:=z_{N \times r}(x)$ is a $N$ by $r$ matrix representing a point in $G(r, N) I_{N \times N}$ is the identity matrix and $V$ is the volume of $X$. We call the equation above the balance equation.

We have seen in $[\mathrm{W}]$ that the balance equation is a moment map equation. To relate it to Atiyah-Bott's moment map description of Hermitian-Einstein equation, we need a local version of above theorem. First, let us introduce Hermitian metrics $h$ and $H$ on $\mathcal{O}_{X}(1)$ and $\mathcal{E}$ respectively, and fix the Kähler form on $X$ to be $\omega:=\frac{\sqrt{-1}}{2 \pi} \operatorname{Ric}(h)$. Let $V$ denote the volume of $(X, \omega)$. Suppose $\left\{S_{1}, \cdots, S_{N}\right\}$ is an orthonormal basis of $H^{0}(\mathcal{E}(k))$ with respect to the induced $L^{2}$-metric, then the Bergman kernel $B_{k}$ of $\mathcal{E}(k)$ is defined by the following:

$$
B_{k}(x):=\sum_{\alpha=1}^{N}\left\langle\cdot, S_{\alpha}(x)\right\rangle S_{\alpha}(x): \mathcal{E}_{x} \rightarrow \mathcal{E}_{x} .
$$

Clearly, it is independent of the choice of orthonormal basis. Now the local form of Theorem 1.1 can be stated as following

Corollary 1.1. $\mathcal{E}$ is Gieseker stable if and only if there is a number $k_{0}$ such that for any $k>k_{0}$, we can find a metric $H^{(k)}$ which we will call the balanced metric on $\mathcal{E}(k)$, such that the Bergman Kernel satisfies the equation

$$
B_{k} \equiv \frac{\chi(k)}{V r} \cdot I_{\mathcal{E}}
$$

where $I_{\mathcal{E}}$ is the identity bundle morphism and $\chi(k)$ is the Hilbert polynomial of $\mathcal{E}$ with respect to the polarization $\mathcal{O}_{X}(1)$.

From the above corollary we deduce that if $\mathcal{E}$ is Gieseker stable then for each $k>>1$ there is a balanced metric $H^{(k)}$ on $\mathcal{E}(k)$. Hence we will have a sequence of Hermitian metric $H_{k}:=H^{(k)} \otimes h^{-k}$ on $\mathcal{E}$. The main result of this paper is to answer the second question of Donaldson in [D1] by proving the following:

Theorem 1.2. Suppose $\mathcal{E}$ is Gieseker stable. If for $k \rightarrow \infty$ we have $H_{k} \rightarrow$ $H_{\infty}$ in $C^{\infty}$, then metric $H_{\infty}$ solves the following weakly Hermitian-Einstein equation. 


$$
\frac{\sqrt{-1}}{2 \pi} \bigwedge F_{\left(\mathcal{E}, H_{\infty}\right)}+\frac{S(\omega)}{2} \cdot I_{\mathcal{E}}=\left(\frac{\operatorname{deg}(\mathcal{E})}{V r}+\frac{\bar{s}}{2}\right) \cdot I_{\mathcal{E}}
$$

where $\bigwedge F_{\left(\mathcal{E}, H_{\infty}\right)}$ is the contraction of curvature form of $\mathcal{E}$ with respect to $\omega$, $S(\omega)$ is the scalar curvature of $X$ and $\bar{s}:=\frac{1}{V} \int_{X} S \frac{\omega^{n}}{n !}$.

Conversely, suppose there is a Hermitian metric $H_{\infty}$ on $\mathcal{E}$ solving (2) then $H_{k} \rightarrow H_{\infty}$ in $C^{r}$ norm for any $r$.

It follows from Proposition IV.2.4 in [K] that the equation (2) is equivalent to the original Hermitian-Einstein equation up to a conformal change of the metric on $\mathcal{E}$. By [UY], we deduce that if $\mathcal{E}$ is irreducible then the solvability of equation (2) is equivalent to $\mathcal{E}$ being Mumford stable.

Remark 1.1. As we have seen from $[\mathrm{AB}]$ and $[\mathrm{W}]$ that both equation (1) and (2) are moment map equations, if we combine this with our knowledge on Bergman kernel expansion(see Appendix)then we see that Theorem 1.2 and Corollary 1.1 precisely describe how finite dimensional moment maps approximate the infinite dimensional one. Since it is well known that there are holomorphic vector bundles which are Gieseker stable but Mumford unstable(see for example [W1] and [OSS]), $H_{k}$ do NOT converge in general. This suggests that we probably should not expect the convergence of balanced metrics in [D2] for projective embedding of manifolds in general either.

The paper is organized as following. In section 2, we first put the problem into the framework of moment map geometry. In particular, we present two equivalent approaches, one is infinite dimensional and the other is finite dimensional. Both approaches rely on the analytical estimate presented in section 3, which is essentially due to Donaldson. In section 4, we first construct approximating solutions to the balance equation (1) and then find the genuine solutions nearby, thus prove the main result.

Acknowledgements: The author is very grateful to Jun Li and Conan Leung for many helpful suggestions, to Richard Thomas and Xiaonan Ma for encouraging him to publish this paper. Also the author want to thank the referee for his careful reading of the manuscript and offering many very helpful suggestion. Finally, this paper was a part of author's Ph.D. thesis, he wants to thank his thesis advisor S.-T.Yau for his guidance and encouragement over the years. 


\section{Moment map geometry.}

In this section we will present the moment map description of the equations involved in Theorem 1.1 and Theorem 1.2. There are two different way of doing this, one is infinite dimensional which we follow Donaldson's work [D1] closely and the other is finite dimensional which is along the line of description in $[\mathrm{W}]$. Although they are equivalent, each one has its own advantage the first approach make the quantity we need to estimate more transparent while the second one is simpler and insensible to the singularities of $\mathcal{E}$.

\subsection{Infinite dimensional picture.}

Let us start from a simple example which we will use later.

Example 2.1. Let $\left(\mathbb{V}, J, \Omega_{\mathbb{V}}\right)$ be a symplectic vector space with complex structure $J$ such that $\Omega$ is $J$-compatible, that is $\Omega_{\mathbb{V}}(J \cdot, J \cdot)=\Omega_{\mathbb{V}}(\cdot, \cdot)$ and $\langle\cdot, \cdot\rangle_{\mathbb{V}}:=\Omega_{\mathbb{V}}(J \cdot, \cdot)-\sqrt{-1} \Omega_{\mathbb{V}}(\cdot, \cdot)$ be the corresponding Hermitian metric. Now let us consider

$$
\mathbb{V}^{N}:=\prod_{\alpha=1}^{N} \mathbb{V}=\mathbb{V} \times \cdots \times \mathbb{V}
$$

with the induced Hermitian inner product given as following

$$
\langle S, T\rangle_{\mathbb{V}^{N}}:=\sum_{\alpha=1}^{N}\left\langle S_{\alpha}, T_{\alpha}\right\rangle_{\mathbb{V}}
$$

where $S:=\left(S_{1}, \cdots, S_{N}\right), T:=\left(T_{1}, \cdots, T_{N}\right) \in \mathbb{V}^{N}$. There is an natural $U(N)$ action on $\mathbb{V}^{N}$ given by

$$
A \cdot S:=A \cdot\left(S_{1}, \cdots, S_{N}\right)=\left(\sigma_{1}, \cdots, \sigma_{N}\right)
$$

where $\sigma_{\alpha}=A_{\beta \alpha} S_{\beta}$. It is a Hamiltonian action with respect to the symplectic form given as the following

$$
\Omega_{\mathbb{V} N}(S, T):=\sum_{\alpha=1}^{N} \Omega_{\mathbb{V}}\left(S_{\alpha}, T_{\alpha}\right) .
$$

Claim. The moment map of this Hamiltonian action is given by the induced infinitesimal action is given by

$$
\nu(S)=\frac{\sqrt{-1}}{2}\left\langle S_{\alpha}, S_{\beta}\right\rangle_{\mathbb{V}}
$$


In particular, if we use $S U(N)$ action we have the moment map is given by

$$
\nu(S)=\frac{\sqrt{-1}}{2}\left(\left\langle S_{\alpha}, S_{\beta}\right\rangle_{\mathbb{V}}-\frac{\sum_{\alpha=1}^{N}\left\|S_{\alpha}\right\|_{\mathbb{V}}^{2}}{N} \delta_{\alpha \beta}\right),
$$

Proof. We will just do the case $A \in \mathfrak{u}(N)$. A induces an infinitesimal action

$$
\tilde{A}=\left.\frac{d}{d t}\right|_{t=0} \exp (t A) \cdot\left(S_{1}, \cdots, S_{N}\right)=\left(\sigma_{1}, \cdots, \sigma_{N}\right)
$$

where $\sigma_{\alpha}=A_{\beta \alpha} S_{\beta}$. Let $S(t):=\left(S_{1}(t), \cdots, S_{N}(t)\right)$ be a curve in $\mathbb{V}^{N}$ such that $S(0)=S_{0} \in \mathbb{V}^{N}$,

$$
\begin{aligned}
& X:=\left.\frac{d}{d t}\right|_{t=0}\left(S_{1}(t), \cdots, S_{N}(t)\right)=\left(\dot{S}_{1}, \cdots, \dot{S}_{N}\right) \\
&\langle X \cdot \nu(S), A\rangle_{\mathfrak{u}(N)}=-\sum_{\alpha, \beta} \frac{\sqrt{-1}}{2} X\left\langle S_{\alpha}, S_{\beta}\right\rangle_{\mathbb{V}} \cdot A_{\alpha \beta} \\
&=-\sum_{\alpha, \beta} \frac{\sqrt{-1}}{2}\left(\left\langle\dot{S}_{\alpha}, S_{\beta}\right\rangle_{\mathbb{V}}+\left\langle S_{\alpha}, \dot{S}_{\beta}\right\rangle_{\mathbb{V}}\right) \cdot A_{\alpha \beta} \\
&=-\frac{\sqrt{-1}}{2} \sum_{\alpha, \beta}\left(\left\langle\dot{S}_{\alpha}, \bar{A}_{\alpha \beta} S_{\beta}\right\rangle_{\mathbb{V}}+\left\langle A_{\alpha \beta} S_{\alpha}, \dot{S}_{\beta}\right\rangle_{\mathbb{V}}\right) \\
&=-\frac{\sqrt{-1}}{2} \sum_{\alpha, \beta}\left(-\left\langle\dot{S}_{\alpha}, A_{\beta \alpha} S_{\beta}\right\rangle_{\mathbb{V}}+\overline{\left\langle\dot{S}_{\beta}, A_{\alpha \beta} S_{\alpha}\right\rangle_{\mathbb{V}}}\right) \\
&=-\frac{\sqrt{-1}}{2} \sum_{\alpha}\left(-\left\langle\dot{S}_{\alpha}, \sigma_{\alpha}\right\rangle_{\mathbb{V}}+\overline{\left\langle\dot{S}_{\alpha}, \sigma_{\alpha}\right\rangle_{\mathbb{V}}}\right) \\
&=\Omega_{\mathbb{V} N}(X, \tilde{A})
\end{aligned}
$$

A simple application of above setting is that the moment map for the standard $U(N)$ action on $\left(\mathbb{C}^{N}, \frac{\sqrt{-1}}{2} d z_{\lambda} \wedge d \bar{z}_{\lambda}\right)$ is given by

$$
\nu(z)=\frac{\sqrt{-1}}{2} z z^{*}
$$

Consider the family version of the example above. Let $\mathcal{E} \rightarrow X$ be a rank $r$ Hermitian vector bundle over $X$. Then the natural symplectic form on the space of sections $\Gamma(\mathcal{E})$ is

$$
\Omega\left(S_{1}, S_{2}\right):=\operatorname{Re}\left(J S_{1}, S_{2}\right)=\operatorname{Re} \int_{X}\left\langle J S_{1}, S_{2}\right\rangle \frac{\omega^{n}}{n !}
$$


and the moment map for the action of the $U(r)$-gauge group $\mathcal{G}$ on $\Gamma(\mathcal{E})$ is given by

$$
\mu(S)=\frac{i}{2} S S^{*} \frac{\omega^{n}}{n !} .
$$

Now let us bring in the holomorphic structures on $\mathcal{E}$. Giving $\mathcal{E}$ a holomorphic structure is equivalent to defining a $\bar{\partial}$-operator,

$$
\bar{\partial}: \Omega^{0}(\mathcal{E}) \longrightarrow \Omega^{0,1}(\mathcal{E}), \bar{\partial}(f \cdot S)=\bar{\partial} f \cdot S+f \cdot \bar{\partial} S,
$$

whose natural prolongation satisfies $\bar{\partial}^{2}=0$. Two $\bar{\partial}$-operators give isomorphic holomorphic structures if and only if they are conjugate by an element in the gauge group $\mathcal{G}$. We say that a connection $A$ is compatible with a holomorphic structure if the $(0,1)$ component of the covariant derivative

$$
d_{A}=\partial_{A}+\bar{\partial}_{A}: \Omega^{0}(\mathcal{E}) \longrightarrow \Omega^{1}(\mathcal{E})=\Omega^{1,0}(\mathcal{E}) \oplus \Omega^{0,1}(\mathcal{E}),
$$

is the $\bar{\partial}$-operator above. In particular, there is a unique connection compatible with both holomorphic and Hermitian structures. If $H=\langle\cdot, \cdot\rangle_{1}$, $K=\langle\cdot, \cdot\rangle_{2}$ are two metrics with

$$
\langle S, T\rangle_{2}=\langle h S, T\rangle_{1}
$$

for an element $h=H^{-1} K \in \Gamma(\operatorname{End}(\mathcal{E}))$ then the corresponding connections are related in the following way

$$
\begin{gathered}
\bar{\partial}_{2}=\bar{\partial}_{1}, \\
\partial_{2}=h^{-1} \circ \partial_{1} \circ h=\partial_{1}+h^{-1} \partial h,
\end{gathered}
$$

and the curvature forms $F_{1}, F_{2} \in \Omega^{1,1}(\operatorname{End}(\mathcal{E}))$ are related by the following equation

$$
F_{2}=F_{1}+\bar{\partial}_{1}\left(h^{-1} \partial h\right) .
$$

Let $\mathcal{A}$ denote the space of connections on $\mathcal{E}$. Then any unitary connection in the subset

$$
\mathcal{A}^{(1,1)}=\left\{A \in \mathcal{A} \mid F_{A}^{(0,2)}=F_{A}^{(2,0)}=0\right\} \subset \mathcal{A}
$$

defines a holomorphic structure on $E$. The complex gauge group $\mathcal{G}^{\mathbb{C}}$ acts on $\mathcal{A}^{(1,1)}$ by

$$
\begin{gathered}
\bar{\partial}_{g(A)}=g \circ \bar{\partial}_{A} \circ g^{-1}, \\
\partial_{g(A)}=\left(g^{*}\right)^{-1} \circ \partial_{A} \circ g^{*}, \text { for } A \in \mathcal{A}^{(1,1)}, g \in \mathcal{G}^{\mathbb{C}},
\end{gathered}
$$


extending the action of the unitary gauge group

$$
\mathcal{G}=\left\{g \in \mathcal{G}^{\mathbb{C}} \mid h(g):=g^{*} g=1\right\}
$$

Notice that $\mathcal{A}^{(1,1)}$ has a natural symplectic structure (c.f. $[\mathrm{AB}]$ )

$$
\tilde{\Omega}\left(\alpha_{1}, \alpha_{2}\right):=\int_{X} \operatorname{Tr}\left(\alpha_{1} \wedge \alpha_{2}\right) \frac{\omega^{n-1}}{(n-1) !},
$$

and the moment map for the action of gauge group $\mathcal{G}$ is given by

$$
\tilde{\mu}(A)=F_{A} \cdot \frac{\omega^{n}}{n !} .
$$

Our main interest is exactly the relation between these $\mu$ and $\tilde{\mu}$. Now since the action of the group $\mathcal{G}$ on $\Gamma(\mathcal{E})$ does not preserve the set of holomorphic sections for a fixed connection $A \in \mathcal{A}^{(1,1)}$, it is natural to consider the diagonal action of $\mathcal{G}$ on the space

$$
\mathcal{H}:=\overbrace{\Gamma(\mathcal{E}(k)) \times \cdots \times \Gamma(\mathcal{E}(k))}^{N} \times \mathcal{A}^{(1,1)}
$$

where $N=\operatorname{dim} H^{0}(X, \mathcal{E}(k)) .{ }^{1}$ Let $\mathcal{H}_{0}$ be the subspace of $\mathcal{H}$ consisting of

$$
\left(S_{1}, \cdots, S_{N} ; A\right)
$$

such that $\bar{\partial}_{A} S_{\alpha}=0, \forall \alpha$ and $S_{\alpha}$ 's are linearly independent. Clearly $\mathcal{G}$ action preserves $\mathcal{H}_{0}$, since $\bar{\partial}_{g(A)} g S_{\alpha}=g \circ \bar{\partial}_{A} \circ g^{-1}\left(g S_{\alpha}\right)=g\left(\bar{\partial}_{A} S_{\alpha}\right)=0$. So the $\mathcal{G}^{\mathbb{C}}$-orbit are equivalence classes of "holomorphic vector bundles and a basis of holomorphic sections". Now if we look at the diagram

$$
\begin{gathered}
\mathcal{H}_{0} \hookrightarrow \Gamma(\mathcal{E}(k)) \times \cdots \times \Gamma(\mathcal{E}(k)) \times \mathcal{A}^{(1,1)} \stackrel{\pi_{2}}{\longrightarrow} \mathcal{A}^{(1,1)} \\
\qquad \pi_{1} \\
\Gamma(\mathcal{E}(k)) \times \cdots \times \Gamma(\mathcal{E}(k))
\end{gathered}
$$

then we will see that the restriction of $\pi_{1}$ to $\mathcal{H}_{0}$ is injective for sufficient large $k$ at least in a neighborhood of $\mathcal{G}^{\mathbb{C}} \cdot A \subset \mathcal{A}^{1,1}$ (see the footnote). So we

\footnotetext{
${ }^{1}$ In general $N$ depends on $A$. It follows from upper semi-continuity theorem that for $k>>1$ there is an open neighborhood $U_{A}$ of $\mathcal{G}^{\mathbb{C}}$-orbit of $A$ in $\mathcal{A}^{1,1}$ on which $H^{i}(X, \mathcal{E}(k))=0, \forall i>0$ and $\operatorname{dim}^{0}(X, \mathcal{E}(k))$ is constant. This is already enough for our later discussion, since we only care one $\mathcal{G}^{\mathbb{C}}$-orbit.
} 
can pull back Kähler form $\Omega$ on $\Gamma(\mathcal{E}(k))$ to $\mathcal{H}_{0}$. The moment map for the $\mathcal{G}$ action with respect $\Omega$ is given by

$$
\mu_{\mathcal{G}}\left(s_{1}, \cdots, s_{N} ; A\right)=\frac{\sqrt{-1}}{2} \sum_{\alpha} S_{\alpha} S_{\alpha}^{*} \frac{\omega^{n}}{n !},
$$

where $S_{\alpha}^{*}:=\left\langle\cdot, S_{\alpha}\right\rangle$. We will drop the term $\frac{\omega^{n}}{n !}$ under the natural pairing between top forms and functions on $X$.

Notice that although both $\tilde{\mu}$ and $\mu_{\mathcal{G}}$ are EndE valued, $\tilde{\mu}$ depends only on the connection. So we have to divide out the freedom of choice of basis. That is precisely another symmetry on $\mathcal{H}_{0}$, i.e. the action of $S U(N)$ on the basis $\left(S_{1}, \cdots, S_{N}\right)$ and the moment map for this action is given by

$$
\mu_{S U}\left(S_{1}, \cdots, S_{N} ; A\right)=\frac{\sqrt{-1}}{2}\left(\left(S_{\alpha}, S_{\beta}\right)-\frac{\sum\left\|S_{\alpha}\right\|_{L^{2}}^{2}}{N} \delta_{\alpha \beta}\right) .
$$

So the finite dimensional candidates for $\tilde{\mu}=a$ is

$$
\mu_{S U}=0 \text { and } \mu_{\mathcal{G}}=a .
$$

which is exactly the balance equation (1). Or another way of saying that is the finite dimensional model is the double symplectic quotient

$$
\mathcal{H}_{0} / /(\mathcal{G} \times S U(N))=\frac{\mu_{\mathcal{G}}^{-1}(a) \cap \mu_{S U}^{-1}(0)}{\mathcal{G} \times S U(N)}
$$

\subsection{Finite dimensional picture.}

Now we take the finite dimensional point of view, there is only one group involved and it also works for sheaves which are not necessarily locally free. First let us recall from [W] that $\operatorname{Map}^{\text {holo }}(X, G(r, N))^{2}$ is the space of holomorphic maps from $X$ to the Grassmanian $G(r, N)$ and we have the following diagram

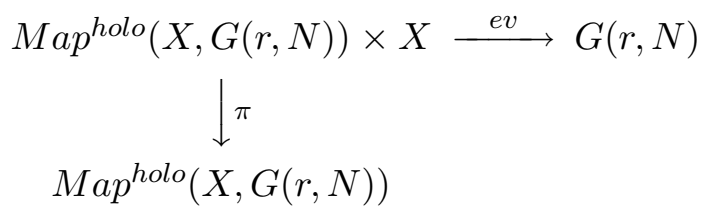

\footnotetext{
${ }^{2}$ Strictly speaking we should use Quot-scheme instead, but since we only care about one $S L(N)$-orbit in Quot-scheme we will not distinguish them.
} 
where $e v(f, x)=f(x), \pi(f, x)=f$ and $\omega_{G}$ is the Fubini-Study Kähler form on $G(r, N)$. The symplectic form on $\operatorname{Map}^{\text {holo }}(X, G(r, N))$ is given by

$$
\Omega=\pi_{*}\left(e v^{*} \omega_{G} \wedge \frac{\omega^{n}}{n !}\right) .
$$

Now the $S U(N)$ action on $G(r, N)$ induces an action on $M a p^{\text {holo }}(X, G(r, N))$ with moment map given by

$$
\mu_{M a p^{h o l o}(X, G(r, N))}=\int_{X} \mu_{G(r, N)} \frac{\omega^{n}}{n !}
$$

which is exactly the balance equation (1).

To reach our final goal we need another bullet, a finite dimensional implicit function theorem for the moment map. To state the result, let us recall that if we have a compact Lie group $G$ acting on a Kähler manifold $Z$ with moment map $\nu: Z \rightarrow \mathfrak{g}$ then at each point $z \in Z$ we have the infinitesimal action

$$
\sigma_{z}: \mathfrak{g} \longrightarrow T Z_{z}
$$

Now we define an endomorphism of $\mathfrak{g}$ by

$$
Q_{z}=\sigma_{z}^{*} \sigma_{z}
$$

where the adjoint is formed using the metrics on $\mathfrak{g}$ and $T Z$. Then we have the following identity

$$
Q_{z}=d \nu_{z} \circ J \circ \sigma_{z}
$$

where is $J$ is almost complex structure on $Z$.

Suppose now that the stabilizers of all points under the $G$-action are discrete, so $Q_{z}$ is invertible for all $z$ in $Z$. We define

$$
\Lambda_{z}:=\max _{\xi \in \mathfrak{g}} \frac{\left\|Q_{z}^{-1} \xi\right\|}{\|\xi\|}
$$

where the norms are defined using the invariant inner product on $\mathfrak{g}$. In order to find a solution to the equation $\nu=0$ we have the following result.

Proposition 2.1. ([D2] Proposition 17.) Suppose given $z_{0} \in Z$ and real numbers $\lambda, \delta$ such that $\Lambda_{z} \leq \lambda$ for all $z=e^{i \xi} z_{0}, \xi \in \mathfrak{g}$ with $|\xi| \leq \delta$. Suppose that $\lambda\left|\nu\left(z_{0}\right)\right|<\delta$. Then there is point $w=e^{i \eta} z_{0}, \eta \in \mathfrak{g}$ with $\nu(w)=0$, where $|\eta| \leq \lambda\left|\nu\left(z_{0}\right)\right|$.

Remark 2.1. Notice that the proposition gives us not only the existence but also the distance between the honest solution and the approximate one. 
To adapt the proposition to the double symplectic quotient setting (10) we need to treat the case that $Z=W / / H$, and the action of $G$ on $Z$ is induced by an action of $G \times H$ on the Kähler manifold $W$. For each point $w \in W$ the infinitesimal action gives

$$
\sigma_{G, w}: \mathfrak{g} \rightarrow T W_{w}, \sigma_{H, w}: \mathfrak{h} \rightarrow T W_{w}
$$

In this variation $\Lambda_{z}$ can be computed via the following.

Lemma 2.1. ([D2] Lemma 18.) Let $z \in W / / H$ be represented by a point $w \in W$. Then for $\xi \in \mathfrak{g}$, the endomorphism $Q_{z}$ of $\mathfrak{g}$ associated to the $G$ action on $W / / H$ satisfies

$$
\left\langle Q_{z} \xi, \xi\right\rangle=\left|\pi\left(\sigma_{G, w} \xi\right)\right|^{2}
$$

where $\pi: T W_{w} \rightarrow T W_{w}$ is the orthogonal projection to $\operatorname{Im}\left(\sigma_{H, w}\right)^{\perp}$. In particular

$$
\Lambda_{z}=\left(\min _{\xi \in \mathfrak{g}} \frac{\left|\pi\left(\sigma_{G, w} \xi\right)\right|}{|\xi|}\right)^{2}
$$

\section{Main estimate.}

Our task of this whole section to estimating $\Lambda_{z}$.

\subsection{Preparations.}

We first derive the formulae that are needed for the estimates. Consider the action of the group $S U(N)$ on the symplectic quotient

$$
Z:=\mathcal{H}_{0} / / \mathcal{G}
$$

We fix our attention on a single orbit of the complex group $S L(N, \mathbb{C})$; that is, we fix attention on a given holomorphic vector bundle $\mathcal{E}(k) \rightarrow X$. Our main goal is to solve the equation $\mu_{S U}(z)=0$ in the given complex orbit. We want to apply the Proposition 2.1, and to do this we need to estimate $\Lambda_{z}$, for $z \in Z$, using the formula of Lemma 2.1. We need to keep in mind two points of view: an element of the orbit is represented by a pair $\left(S_{1}, \cdots, S_{N} ; A\right)$ with $\sum S_{\alpha} S_{\alpha}^{*}=I$, or equivalently by an embedding, still denoted by $z: X \hookrightarrow$ $G(r, N)$ with induced Fubini-Study metric. Given a matrix $A=\left(a_{\alpha \beta}\right) \in$ $\sqrt{-1} \mathfrak{s u}(N)$ we write

$$
\sigma_{\alpha}=\sum_{\alpha} a_{\alpha \beta} S_{\beta}
$$


To apply Lemma 2.1, we need to find the orthogonal projection of

$$
\underline{\sigma}:=\left(\sigma_{1}, \cdots, \sigma_{N}\right)
$$

in the Hilbert space $\Gamma(\mathcal{E}(k))^{N}$, to the orthogonal complement of the subspace

$$
P:=\left\{\left(g S_{1}, \cdots, g S_{N}\right) \mid g \in \operatorname{End}_{\mathbb{C}} \mathcal{E}\right\} .
$$

Proposition 3.1. Given $S_{\alpha}$ and $A=\left(a_{\alpha \beta}\right) \in \sqrt{-1} \mathfrak{s u}(N)$, define $H_{A} \in$ $\operatorname{End}_{\mathbb{C}} \mathcal{E}$ by

$$
H_{A}:=\sum_{\alpha, \beta} a_{\beta \alpha} S_{\alpha} S_{\beta}^{*}
$$

Then the orthogonal projection of $\underline{\sigma}$ to the subspace $P$ is

$$
\underline{p}:=\left(H_{A} S_{1}, \cdots, H_{A} S_{N}\right)
$$

Proof. We write

$$
\psi_{\alpha}:=H_{A} S_{\alpha}-\sigma_{\alpha}
$$

We have to show that, for any $g \in \operatorname{End}_{\mathbb{C}} \mathcal{E}$

$$
\begin{aligned}
& \sum_{\alpha}\left\langle\psi_{\alpha}, g S_{\alpha}\right\rangle=0 . \\
\sum_{\alpha}\left\langle\psi_{\alpha}, g S_{\alpha}\right\rangle= & \sum_{\alpha}\left\langle\sum_{\gamma, \tau} a_{\tau \gamma} S_{\gamma}\left\langle S_{\alpha}, S_{\tau}\right\rangle-\sum_{\beta} a_{\alpha \beta} S_{\beta}, g S_{\alpha}\right\rangle \\
= & \sum_{\alpha} \sum_{\gamma, \tau}\left\langle a_{\tau \gamma} S_{\gamma}, g S_{\alpha}\left\langle S_{\tau}, S_{\alpha}\right\rangle\right\rangle-\left\langle\sum_{\alpha, \beta} a_{\alpha \beta} S_{\beta}, g S_{\alpha}\right\rangle \\
= & \sum_{\gamma, \tau} \sum_{\alpha}\left\langle a_{\tau \gamma} S_{\gamma}, g S_{\alpha}\left\langle S_{\tau}, S_{\alpha}\right\rangle\right\rangle-\left\langle\sum_{\alpha, \beta} a_{\alpha \beta} S_{\beta}, g S_{\alpha}\right\rangle \\
= & \sum_{\gamma, \tau}\left\langle a_{\tau \gamma} S_{\gamma}, g \sum_{\alpha} S_{\alpha}\left\langle S_{\tau}, S_{\alpha}\right\rangle\right\rangle-\left\langle\sum_{\alpha, \beta} a_{\alpha \beta} S_{\beta}, g S_{\alpha}\right\rangle \\
= & \sum_{\gamma, \tau}\left\langle a_{\tau \gamma} S_{\gamma}, g S_{\tau}\right\rangle-\left\langle\sum_{\alpha, \beta} a_{\alpha \beta} S_{\beta}, g S_{\alpha}\right\rangle \\
= & 0 .
\end{aligned}
$$

Note that in the fifth equality follows from the fact that

$$
\sum_{\alpha} S_{\alpha} S_{\alpha}^{*}=\sum_{\alpha} S_{\alpha}\left\langle\cdot, S_{\alpha}\right\rangle=I
$$


Although the space $\mathcal{H}_{0}$ is not smooth in general, we are working on a fixed orbit of $A$ which is smooth. So we are able to apply Lemma 2.1 to obtain that

$$
\Lambda_{z}^{-1}=\min \sum_{\alpha}\left\|\psi_{\alpha}\right\|^{2}=\min \sum_{\alpha}\left\|H_{A} S_{\alpha}-\sum_{\beta} a_{\alpha \beta} S_{\beta}\right\|^{2}
$$

where the minimum runs over $A \in \sqrt{-1} \mathfrak{s u}(N)$ with $\|A\|^{2}=\sum\left|a_{\alpha \beta}\right|^{2}=1$, and $H_{A}$ is defined in terms of $A$ and $S_{\alpha}$ as before. Our task is to find a lower bound for the sum above.

Proposition 3.2. Continuing with the notation above, we have at any fixed point $x \in X$

$$
\sum_{\alpha}\left\|\bar{\partial}_{\mathcal{E}(k)} \psi_{\alpha}\right\|^{2}=\left\|\bar{\partial}_{\operatorname{End} \mathcal{E}(k)} H_{A}\right\|_{o p}^{2}
$$

where $\|\cdot\|_{o p}$ is the operator norm of $H_{A} \in \Gamma(\operatorname{End} \mathcal{E}(k))$ at point $x$.

Proof. First we notice that the statement is unaffected under the $S U(N)$ transformation, so we are free to choose good co-ordinates. Second, recall that $\psi_{\alpha}:=H_{A} S_{\alpha}-\sigma_{\alpha}$, and $\bar{\partial}_{\mathcal{E}(k)} S_{\alpha}=0$, which implies $\bar{\partial}_{\mathcal{E}(k)} \sigma_{\alpha}=0$. We have $\bar{\partial}_{\mathcal{E}(k)} \psi_{\alpha}=\bar{\partial}_{\mathcal{E}(k)}\left(H_{A} S_{\alpha}\right)=\left(\bar{\partial}_{\operatorname{End}(k)} H_{A}\right) S_{\alpha}$. Since $\sum_{\alpha} S_{\alpha} S_{\alpha}^{*}=I, S_{\alpha}$ 's are actually the co-ordinate sections of the universal quotient bundle on $G(r, N)$, which implies that up to a $S U(N)$ transformation we may assume that $\left\{S_{1}, \cdots, S_{r}\right\}$ is an orthonormal bases and $S_{r+1}=\cdots=S_{N}=0$ at the point $x \in X$. With this understood, we have

$$
\sum_{\alpha}\left\|\bar{\partial}_{\mathcal{E}(k)} \psi_{\alpha}\right\|^{2}=\sum_{\alpha}\left\|\left(\bar{\partial}_{\operatorname{End} \mathcal{E}(k)} H_{A}\right) S_{\alpha}\right\|^{2}=\left\|\bar{\partial}_{\operatorname{End} \mathcal{E}(k)} H_{A}\right\|_{o p}^{2}
$$

at every point $x \in X$.

If we take the finite dimensional point of view then we need to consider the action of group $S U(N)$ on $\operatorname{Map}^{\text {holo }}(X, G(r, N))$. In this set up, $\sigma_{z}(A)=$ $X_{A} \in \Gamma\left(X, z^{*} T G(r, N)\right)$ is the vector field induced by the $A$ along $z(X) \subset$ $G(r, N)$ and

$$
\Lambda_{z}^{-1}=\min _{\|A\|=1}\left\|X_{A}\right\|^{2}
$$

The following proposition relates above two moment map approaches to each other. Thus both approaches rely on the same estimates. 


\section{Proposition 3.3.}

$$
\sum_{\alpha}\left\|\psi_{\alpha}\right\|^{2}=\pi\left\|X_{A}\right\|^{2}
$$

where

$$
\left\|X_{A}\right\|^{2}:=\int_{X} \omega_{F S}\left(X_{A}, J X_{A}\right) \frac{\omega^{n}}{n !}
$$

Proof. From formula (18) we deduce

$$
\begin{aligned}
& \sum_{\alpha}\left\|\psi_{\alpha}\right\|^{2} \\
= & -\sum_{\alpha}\left\langle\psi_{\alpha}, \sigma_{\alpha}\right\rangle \\
= & -\sum_{\alpha}\left\langle H_{A} S_{\alpha}-\sigma_{\alpha}, \sigma_{\alpha}\right\rangle \\
= & -\sum_{\alpha, \beta, \gamma, \tau} a_{\beta \gamma} a_{\tau \alpha}\left\langle S_{\alpha}, S_{\beta}\right\rangle\left\langle S_{\gamma}, S_{\tau}\right\rangle+\sum_{\alpha, \beta, \gamma} a_{\alpha \beta} a_{\gamma \alpha}\left\langle S_{\beta}, S_{\gamma}\right\rangle
\end{aligned}
$$

On the other hand, a point $z \in G(r, N)$ can be thought as an $N \times r$ matrix. So we have

$$
\begin{aligned}
& \left\|X_{A}\right\|^{2}=\left\|X_{i A}\right\|^{2} \\
= & \omega_{F S}\left(X_{i A}, J X_{i A}\right) \\
= & \left\langle d \mu_{G(r, N)}\left(J X_{i A}\right), i A\right\rangle \\
= & \left.\frac{1}{2 \pi} \frac{d}{d t}\right|_{t=0} \operatorname{Tr}\left(e^{A t} z\left(z^{*} e^{A t} e^{A t} z\right)^{-1} z^{*} e^{A t} \cdot A\right) \\
= & \frac{1}{\pi} \operatorname{Tr}\left(z\left(z^{*} z\right)^{-1} z^{*} A A\right)-\frac{1}{\pi} \operatorname{Tr}\left(z\left(z^{*} z\right)^{-1} z^{*} A z\left(z^{*} z\right)^{-1} z^{*} A\right)
\end{aligned}
$$

the proposition follows from the fact

$$
z\left(z^{*} z\right)^{-1} z^{*}=\left\{\left\langle S_{\alpha}, S_{\beta}\right\rangle_{z}\right\}
$$

\subsection{Estimates of $\Lambda_{z}$.}

Now we are ready to estimate $\Lambda_{z}$. Let us fix any reference metric $H_{0}$ on $\mathcal{E}$. For any given $k$, an orthonormal base of $H^{0}(X, \mathcal{E}(k))$ with respect to the metric $H_{0} \otimes h^{k}$ gives rise to a projective embedding $X \rightarrow G(r, N)$. Let 
$H_{0, k}^{\prime}$ denote the restriction of Fubini-Study metric on $\mathcal{E}(k)$ induced from the embedding then these two metrics are equivalent in the following sense

$$
H_{0} \otimes h^{k} \sim \frac{\chi(k)}{r V} H_{0, k}^{\prime}
$$

Definition 3.1. Given $R>0$ and $r>2$ for fixed $k$ we say that another metric $H$ has $R$-bounded geometry if

1) $H>\frac{1}{R} H_{0, k}^{\prime}$,

2) $\left\|H-H_{0, k}^{\prime}\right\|_{C^{r}}<R$,

where the norm $\|\cdot\|_{C^{r}}$ is the $C^{r}$ norm determined by the metric $H_{0, k}^{\prime}$. Similarly, we say that the basis $\left\{S_{\alpha}\right\}_{\alpha=1}^{N}$ for $H^{0}(\mathcal{E}(k))$ has $R$-bounded geometry if the Hermitian metric $H$ induced from the Fubini-Study metric by the embedding of $X$ in $G(r, N)$ does.

Throughout this section all norms are with respect to the metric $H_{0, k}^{\prime}$ on $\mathcal{E}(k)$ unless we stress explicitly. Notice that, for this metric if we integrate over $X$ then we have

$$
\sum_{\alpha}\left\|S_{\alpha}\right\|_{L^{2}}=r V
$$

we write

$$
\left(S_{\alpha}, S_{\beta}\right)=\frac{r V}{N} \delta_{\alpha \beta}+\eta_{\alpha \beta}
$$

Thus the error matrix $E=\left(\eta_{\alpha \beta}\right)$ is the traceless Hermitian matrix, and $E=0$ if and only if the projective embedding is balanced.

We continue with the notation from Section 3.1, so for any matrix $A=\left(a_{\alpha \beta}\right) \in \sqrt{-1} \mathfrak{s u}(N)$ we define $H_{A} \in \Gamma($ End $\mathcal{E})$ and section $\psi_{\alpha}$ as in Proposition 3.1. We write $\underline{\psi}$ for the vector

$$
\left(\psi_{1}, \cdots, \psi_{N}\right) \in \Gamma(\mathcal{E}(k)) \times \cdots \times \Gamma(\mathcal{E}(k))
$$

so we have

$$
\|\underline{\psi}\|_{L^{2}}^{2}=\sum_{\alpha}\left\|\psi_{\alpha}\right\|_{L^{2}}^{2}
$$

Let us recall two standard norms on the Hermitian matrices: the HilbertSchmidt norm

$$
\|M\|^{2}=: \sum_{\alpha, \beta}\left|M_{\alpha \beta}\right|^{2}
$$

and the operator norm

$$
\|M\|_{o p}=: \max _{|\xi|=1} \frac{|M \xi|}{|\xi|}
$$


then we have the following inequalities, for $N \times N$ Hermitian matrices $M, L$

$$
\begin{gathered}
|\operatorname{Tr}(M L M)| \leq\|M\|^{2}\|L\|_{o p} \\
|\operatorname{Tr}(L M)| \leq \sqrt{N}\|M\|\|L\|_{o p} .
\end{gathered}
$$

In the following discussion, we will use $C$ to denote any constant which only depends on the geometry of $X$ and the fixed background Hermitian metric $H_{0}$ on $\mathcal{E}$ but independent of $k$.

Theorem 3.1. Suppose $\mathcal{E}$ is simple. For any $R$ there is a constant $C:=$ $C\left(R, H_{0}, r, h\right)$ and $\epsilon:=\epsilon\left(R, H_{0}, r, h\right)<\frac{1}{2}$ so that, for any $k$ if a basis $\left\{S_{\alpha}\right\}$ 's of $H^{0}(\mathcal{E}(k))$ has $R$-bounded geometry and with $k^{n}\|E\|_{o p}<\epsilon$. Then

$$
\|A\| \leq C k^{\frac{3+5 n}{2}}\|\underline{\psi}\|_{L^{2}}
$$

holds for any traceless Hermitian matrix $A$,

Let $\left\{S_{\alpha}\right\}$ be a basis embedding $X$ into $G(r, N)$. By Section 2 this gives rise to two points, one in $Z=\mathcal{H}_{0} / / \mathcal{G}$ and the other in $\operatorname{Map}(X, G(r, N))$. If we denote both points by $z$ then $\Lambda_{z}$ 's are defined by (20) or (23) respectively. Theorem 3.1 above and Proposition 3.3 then imply the following

Corollary 3.1. Suppose $\left\{S_{\alpha}\right\}$ satisfying the hypotheses of Theorem 3.1, Then both $\Lambda_{z}$ 's satisfy

$$
\Lambda_{z} \leq C k^{3+5 n}
$$

The analytical estimates required to prove Theorem 3.1 are summed up in the following:

Proposition 3.4. Suppose $\mathcal{E}$ is simple. Then there are constants $C^{\prime} s$ and $\epsilon$ which depend on $R, H_{0}$ and $\mathcal{E}$ but independent of $k$ so that for all $k$ sufficiently large, if the basis $\left\{S_{\alpha}\right\}$ of $H^{0}(\mathcal{E}(k))$ has $R$-bounded geometry we have

i)

$$
\left\|\bar{\partial} H_{A}\right\|_{o p-L^{2}}^{2} \leq C k^{\frac{3+3 n}{2}}\|\underline{\psi}\|_{L^{2}}\|A\|
$$

ii)

$$
\left\|H_{A}\right\|_{o p-L^{2}}^{2} \leq C\left\|\bar{\partial} H_{A}\right\|_{o p-L^{2}}^{2}+\frac{N}{V}\|E\|_{o p}^{2}\|A\|^{2},
$$

Moreover, if we assume further that $k^{n}\|E\|_{o p}<\epsilon$ then iii)

$$
\|A\|^{2} \leq C k^{n}\left(\left\|H_{A}\right\|_{o p-L^{2}}^{2}+\|\underline{\psi}\|_{L^{2}}^{2}\right)
$$


where $\|\cdot\|_{o p-L^{2}}$ denotes the $L^{2}$-norm corresponding to $\|\cdot\|_{o p}$ on End $\mathcal{E}$.

Proof of Theorem 3.1 .

$$
\begin{aligned}
\|A\|^{2} & \leq C k^{n}\left(\left\|H_{A}\right\|_{o p-L^{2}}^{2}+\|\underline{\psi}\|_{L^{2}}^{2}\right) \\
& \leq C k^{n}\left(C\left\|\bar{\partial} H_{A}\right\|_{o p-L^{2}}^{2}+\frac{N}{V}\|E\|_{o p}^{2}\|A\|^{2}+\|\underline{\psi}\|_{L^{2}}^{2}\right) \\
& \leq C k^{n}\left(C k^{\frac{3+3 n}{2}}\|\underline{\psi}\|_{L^{2}}\|A\|+\frac{N}{V}\|E\|_{o p}^{2}\|A\|^{2}+\|\underline{\psi}\|_{L^{2}}^{2}\right) \\
& \leq C\left(k^{\frac{3+5 n}{2}}\|\underline{\psi}\|_{L^{2}}\|A\|+k^{2 n}\|E\|_{o p}^{2}\|A\|^{2}+k^{n}\|\underline{\psi}\|_{L^{2}}^{2}\right) .
\end{aligned}
$$

By the assumption on $\|E\|_{o p} \leq \frac{\epsilon}{k^{n}}$, we take an $\epsilon:=\epsilon\left(R, H_{0}, r, h\right)$ such that $\epsilon C<\frac{1}{2} \Rightarrow C k^{2 n}\|E\|_{o p}^{2}<\frac{1}{2}$. So we have

$$
\|A\|^{2} \leq C\left(k^{\frac{3+5 n}{2}}\|\underline{\psi}\|_{L^{2}}\|A\|+k^{n}\|\underline{\psi}\|_{L^{2}}^{2}\right)
$$

Now we have two cases to deal with, either

$$
k^{\frac{3+5 n}{2}}\|\underline{\psi}\|_{L^{2}}\|A\| \leq k^{n}\|\underline{\psi}\|_{L^{2}}^{2}
$$

clearly we will have the $\|A\|^{2} \leq C k^{n}\|\underline{\psi}\|_{L^{2}}^{2}$, or

$$
k^{\frac{3+5 n}{2}}\|\underline{\psi}\|_{L^{2}}\|A\|>k^{n}\|\underline{\psi}\|_{L^{2}}^{2}
$$

again we have

$$
\|A\|^{2} \leq C k^{\frac{3+5 n}{2}}\|\underline{\psi}\|_{L^{2}}\|A\|
$$

which gives us $\|A\| \leq C k^{\frac{3+5 n}{2}}\|\underline{\psi}\|_{L^{2}}$.

Before we prove the Proposition 3.4, let us begin with the following estimate.

Lemma 3.1. Under the hypotheses of Theorem 3.1, there is a constant $C$ such that for any integer $j$

i)

$$
\sum_{\alpha}\left\|\nabla^{j} S_{\alpha}\right\|^{2} \leq C k^{j+n}
$$

ii)

$$
\left\|\nabla^{j} H_{A}\right\|_{L^{2}}^{2} \leq C k^{j+2 n}\|A\|^{2}
$$

at each point of $X$. 
Proof. Let us start with the following toy model. Let

$$
\left(L=\underline{\mathbb{C}}, e^{-|z|^{2}}\right) \rightarrow\left(\mathbb{C}^{n}, \omega_{0}=\sum_{\alpha} \frac{\sqrt{-1}}{2} d z_{\alpha} \wedge d \bar{z}_{\alpha}\right)
$$

be the trivial line bundle with Hermitian metric $h=e^{-|z|^{2}}$ over $\mathbb{C}^{n}$. Now, let $S \equiv 1$ be the global holomorphic section. If we consider line bundle $L^{\otimes k} \rightarrow \mathbb{C}^{n}$ then $\|\bar{\partial} \partial S(0)\|_{h^{k}}=k$, on the other hand

$$
\begin{aligned}
\|S\|_{L^{2}}^{2} & =\int_{\mathbb{C}^{n}} e^{-k|z|^{2}} \omega_{0}^{n} \\
& =\frac{1}{k^{n}} \int_{\mathbb{C}^{n}} e^{-k|z|^{2}}\left(k \omega_{0}\right)^{n}=\left(\frac{\pi}{k}\right)^{n} .
\end{aligned}
$$

In conclusion, we have

$$
\left\|\nabla^{j} S(0)\right\|_{h^{k}}^{2} \leq C k^{j+n}\|S\|_{L^{2}}^{2} .
$$

In general, fix a point $x \in X$ and a geodesic ball $B \subset X$ centered at $x$ over which $\mathcal{E}$ is trivial. Since $H$ is $R$-bounded, by using the quasi-isometry between Hermitian vector bundles $\left(\left.\mathcal{E}(k)\right|_{B}, H_{0} \otimes h^{k}\right)$ and $\left(\underline{\mathbb{C}}^{n}, e^{-k|z|^{2}}\right)$ we deduce for any holomorphic section $S$ of $\mathcal{E}(k)$

$$
\left\|\nabla^{j} S(x)\right\|_{H_{0} \otimes h^{k}}^{2} \leq C k^{j+n} \int_{B(x)}\|S\|_{H_{0} \otimes h^{k}}^{2} d V \leq C k^{j+n} \int_{X}\|S\|_{H_{0} \otimes h^{k}}^{2} d V .
$$

which implies

$$
\left\|\nabla^{j} S(x)\right\|^{2} \leq C k^{j+n} \int_{B(x)}\|S\|^{2} d V \leq C k^{j+n} \int_{X}\|S\|^{2} d V .
$$

Since $X$ is compact, we can choose $C$ so that it depend only on $X,\left(\mathcal{E}, H_{0}\right), R$ and $h$ but not on $k$ and $x$. Summing over $\alpha$ we have

$$
\sum_{\alpha}\left\|\nabla^{j} S_{\alpha}\right\|^{2} \leq C k^{j+n} \int_{X} \sum_{\alpha}\left\|S_{\alpha}\right\|^{2}=C k^{j+n} r V
$$

since $\operatorname{Tr}\left(\sum_{\alpha} S_{\alpha} S_{\alpha}^{*}\right)=r$ at each point.

To prove ii), we just apply the part i).

$$
\begin{aligned}
\left\|\nabla^{j} H_{A}\right\|_{L^{2}}^{2} & =\left\|\sum_{\alpha, \beta} a_{\alpha \beta} \nabla^{j}\left(S_{\beta} S_{\alpha}^{*}\right)\right\|_{L^{2}}^{2} \\
& \leq C \sum_{\alpha, \beta} \sum_{l}\left|a_{\alpha \beta}\right|^{2}\left\|\nabla^{l} S_{\beta}\right\|_{L^{2}}^{2}\left\|\nabla^{j-l} S_{\alpha}^{*}\right\|_{L^{2}}^{2} \\
& \leq C k^{j+2 n}\|A\|^{2} .
\end{aligned}
$$


Proof of Proposition 3.4. Recall from Proposition 3.2 we have

$$
\sum_{\alpha}\left\|\bar{\partial} \psi_{\alpha}\right\|^{2}=\|\bar{\partial} H\|_{o p}^{2}
$$

so

$$
\begin{aligned}
\left\|\bar{\partial}_{\mathcal{E}(k)} H\right\|_{o p-L^{2}}^{2} & =\sum_{\alpha}\left\|\bar{\partial}_{\mathcal{E}(k)} \psi_{\alpha}\right\|_{L^{2}}^{2} \leq\left(\sum_{\alpha}\left\|\Delta_{\mathcal{E}(k)} \psi_{\alpha}\right\|_{L^{2}}^{2} \sum_{\alpha}\left\|\psi_{\alpha}\right\|_{L^{2}}^{2}\right)^{1 / 2} \\
& =\left(\sum_{\alpha}\left\|\Delta_{\mathcal{E}(k)} \psi_{\alpha}\right\|_{L^{2}}^{2}\right)^{1 / 2} \sum_{\alpha}\|\underline{\psi}\|_{L^{2}}
\end{aligned}
$$

Now, for each $\alpha$, we have

$$
\Delta \psi_{\alpha}=\Delta\left(H_{A} S_{\alpha}\right)=\Delta\left(H_{A}\right) S_{\alpha}+2 \nabla H_{A} \cdot \nabla S_{\alpha} .
$$

Summing over $\alpha$ using the first part of Lemma 3.1, integrating over $X$ we have

$$
\sum_{\alpha}\left\|\Delta \psi_{\alpha}\right\|_{L^{2}}^{2} \leq C k^{1+n}\left(\left\|\nabla^{2} H_{A}\right\|_{L^{2}}^{2}+\left\|\nabla H_{A}\right\|_{L^{2}}^{2}\right) \leq C k^{3+3 n}\|A\|^{2},
$$

using the 2nd inequality of Lemma 3.1. Thus we have established the first inequality of Proposition 3.4.

To prove ii), first we have the following inequality due to the simpleness of $\mathcal{E}$

$$
\left\|H_{A}\right\|_{L^{2}}^{2} \leq C\left\|\bar{\partial} H_{A}\right\|_{L^{2}}^{2}+\frac{1}{V}\left(\int_{X} \operatorname{Tr}\left(H_{A}\right) \frac{\omega^{n}}{n !}\right)^{2} .
$$

Now Let $\left\{e_{i}\right\}_{i=1}^{r}$ be a local orthonormal frame for $\mathcal{E}$.

$$
\begin{aligned}
\int_{X} \operatorname{Tr} H_{A}(x) d V & =\int_{X} \sum_{i}\left\langle H_{A} e_{i}, e_{i}\right\rangle d V \\
& =\int_{X} \sum_{i}\left\langle\sum_{\gamma, \delta} a_{\gamma \delta} S_{\delta}\left\langle e_{i}, S_{\gamma}\right\rangle, e_{i}\right\rangle d V \\
& =\int_{X} \sum_{\gamma, \delta} a_{\gamma \delta} \sum_{i} S_{\delta}^{i} \bar{S}_{\gamma}^{i} d V \\
& =\int_{X} \sum_{\gamma, \delta} a_{\gamma \delta}\left\langle S_{\delta}, S_{\gamma}\right\rangle d V \\
& =\sum_{\gamma, \delta} a_{\gamma \delta} \overline{\eta_{\gamma \delta}} \\
& \leq \sqrt{N}\|E\|_{o p}\|A\| .
\end{aligned}
$$


To prove iii), recall that we have the orthogonal decomposition on $\Gamma(\mathcal{E}(k))^{N}$

$$
\underline{\sigma}=\underline{\psi}+\underline{p}
$$

so that we have

$$
\|\underline{\sigma}\|_{L^{2}}^{2}=\|\underline{\psi}\|_{L^{2}}^{2}+\|\underline{p}\|_{L^{2}}^{2}
$$

Now

$$
\|\underline{\sigma}\|_{L^{2}}^{2}=\sum_{\alpha, \beta, \gamma} a_{\alpha \beta} a_{\alpha \gamma}\left(S_{\alpha}, S_{\beta}\right)=\frac{V r}{N} \sum_{\alpha, \beta}\left|a_{\alpha \beta}\right|^{2}+\sum_{\alpha, \beta, \gamma} a_{\alpha \beta} \eta_{\beta \gamma} a_{\gamma \alpha} .
$$

By (27), the last term is bounded by $\|E\|_{o p}\|A\|^{2}$, since $\|E\|_{o p} \leq \frac{\epsilon}{k^{n}}$ by hypothesis, we deduce that

$$
\|A\|^{2} \leq C k^{n}\|\underline{\sigma}\|_{L^{2}}^{2}
$$

Now

$$
\|\underline{p}\|_{L^{2}}^{2}=\sum_{\alpha}\left\|H_{A} S_{\alpha}\right\|_{L^{2}}^{2}=\left\|H_{A}\right\|_{o p-L^{2}}^{2}
$$

where for the last identity follows from the proof of Proposition 3.2.

\section{Proof of Theorem 1.2.}

\subsection{Construction of approximating solutions.}

First, we try to construct "nearly balanced" projective embeddings. Recall that for any fixed Hermitian metrics $H$ and $h$ on $\mathcal{E}$ and $\mathcal{O}_{X}(1)$ respectively, the Bergman kernel is defined by

$$
B_{k}(H, h):=\sum_{\alpha} S_{\alpha} S_{\alpha}^{*} \in \Gamma(\operatorname{End} \mathcal{E}(k))
$$

where $\left\{S_{\alpha}\right\}$ is an orthonormal basis of $H^{0}(X, \mathcal{E}(k))$.

Theorem 4.1. Suppose that $E$ is simple and equipped with a Hermitian metric $H_{\infty}$ solving (2). Then there are $\eta_{1}, \eta_{2}, \cdots \in \Gamma($ End $\mathcal{E})$ such that for any $q>0$ there is a constant $C_{q}$ such that the Hermitian metric on $\mathcal{E}$ of the form

$$
H(k):=H_{\infty}\left(1+\sum_{i=1}^{q} \eta_{i} k^{-i}\right)
$$


satisfies

$$
B_{k}(H(k), h)=\frac{\chi(k)}{r V} I+\sigma_{q}(k)
$$

with

$$
\left\|\sigma_{q}(k)\right\|_{C^{r+2}} \leq C_{q} k^{n-q-1}
$$

for all large enough $k$.

Proof. This is a consequence of the Catlin's result [C] and the expansion formula in the Appendix. Recall that

$$
B_{k}(H, h)=k^{n}+A_{1}(H, h) k^{n-1}+\cdots+A_{q}(H, h) k^{n-q}+O\left(k^{n-q-1}\right)
$$

where $A_{p}^{\prime} s$ are polynomials in the curvature tensor of $H \otimes h^{k}$ and its covariant derivatives, and the error term is uniformly bounded in $C^{r+2}$ for all metrics $H$ in a bounded family. Consider the Taylor expansion of the coefficients

$$
A_{p}(H(1+\eta), h)=A_{p}(H, h)+\sum_{l=1}^{q} A_{p, l}(\eta)+O\left(\|\eta\|_{C^{s}}^{q+1}\right)
$$

where $A_{p, l}(\eta)$ is a homogenous polynomial of degree $l$ depending on $H, h$, $\eta$ and its covariant derivatives and $s$ is sufficiently large (depending on $r$ and q). In particular, we have that $A_{0}(H, h) \equiv 1, A_{0, l}(H, h) \equiv 0$, and $A_{1}(H, h)=\frac{\sqrt{-1}}{2 \pi} \bigwedge F_{\mathcal{E}}+\frac{1}{2} S(h) \cdot I$, where $\bigwedge F_{\mathcal{E}}$ is the contraction of curvature form with respect to $\omega$. Thus, for any $\eta_{1}, \eta_{2}, \cdots, \eta_{q} \in \Gamma($ End $\mathcal{E})$, we can write

$$
A_{p}\left(H\left(1+\sum_{i=1}^{q} \eta_{i} k^{-i}\right), h\right)=A_{p}(H, h)+\sum_{l=1}^{q} b_{p, l}(\eta) k^{-l}+O\left(k^{-q-1}\right)
$$

where the $b_{p, l}$ are certain multi-linear expressions in the $\eta_{j}$, and their covariant derivatives, beginning with

$$
b_{p, 1}=A_{p, 1}\left(\eta_{1}\right) .
$$

Thus we get

$$
\begin{aligned}
& B_{k}\left(H_{\infty}\left(1+\sum_{i=1}^{q} \eta_{i} k^{-i}\right)\right) \\
= & \sum_{p=0}^{q} k^{n-q} A_{p}(H, h)+\sum_{p, l=1}^{q} b_{p, l} k^{n-p-l}+O\left(k^{n-q-1}\right) \\
= & k^{n}+k^{n-1} A_{1}+k^{n-2}\left(A_{2}+b_{1,1}\right)+k^{n-3}\left(A_{3}+b_{2,1}+b_{1,2}\right) \\
+ & k^{n-4}\left(A_{4}+b_{3,1}+b_{2,2}+b_{1,3}\right)+\cdots,
\end{aligned}
$$


and for $\chi(k)$ we have

$$
\chi(k)=\chi_{0} k^{n}+\chi_{1} k^{n-1}+\chi_{2} k^{n-2}+\cdots .
$$

Since

$$
\begin{gathered}
b_{1,1}=\frac{\sqrt{-1}}{2 \pi} \bigwedge \bar{\partial} \partial \eta_{1} \\
\int_{X} \operatorname{Tr}\left(A_{2}-\chi_{2}\right) d V=0
\end{gathered}
$$

and $\mathcal{E}$ is simple, there is an unique $\eta_{1} \in H^{0}(\operatorname{End} \mathcal{E})^{\perp} \subset \Gamma($ End $\mathcal{E})$ solving equation .

In general, suppose we have already solved $\eta_{1}, \cdots, \eta_{p-1}$ then the equation for $\eta_{p}$ is

$$
b_{1, p}=\frac{\sqrt{-1}}{2 \pi} \bigwedge \bar{\partial} \partial \eta_{p}=P_{p}
$$

where

$$
P_{p}:=\chi_{p}-A_{l+1}-\sum_{l=0}^{p-1} b_{p-l, l+1}
$$

is determined by $\eta_{1}, \cdots, \eta_{p-1}$. We claim that $\operatorname{Tr} P_{p}$ is a divergence. This is because the coefficient of $k^{n-p}$ in

$$
B_{k}\left(\left(1+\sum_{l=1}^{p-1} \frac{\eta_{l}}{k^{l}}\right) H_{\infty}, h\right)
$$

is

$$
A_{l+1}+\sum_{l=0}^{p-1} b_{p-l, l+1}
$$

and the identity

$$
\int_{X} \operatorname{Tr} B_{k}(H, h) \frac{\omega^{n}}{n !}=\int_{X} \operatorname{Tr} B_{k}\left(\left(1+\sum_{l=1}^{p-1} \frac{\eta_{l}}{k^{l}}\right) H_{\infty}, h\right) \frac{\omega^{n}}{n !}=\chi(k) .
$$

implies

$$
0=\int_{X} \operatorname{Tr}\left(\chi_{p}-A_{l+1}-\sum_{l=0}^{p-1} b_{p-l, l+1}\right) d V=\int_{X} \operatorname{Tr} P_{p} d V,
$$

that is, $\operatorname{Tr} P_{p}$ is a divergence. Because of the simplicity of $\mathcal{E}$, equation (43) has an unique solution in $H^{0}(\operatorname{End} \mathcal{E})^{\perp} \subset \Gamma($ End $\mathcal{E})$. 
Let us fix a positive integer $q$, by Theorem 4.1 we can find a Hermitian metric $H(k)$ so that

$$
B_{k}(H(k), h)=\frac{\chi(k)}{r V}\left(I+\epsilon_{k}\right)
$$

where $\epsilon_{k}=O\left(k^{n-q-1}\right)$ in $C^{r+2}$. We then define a new Hermitian metric $H(k)^{\prime}$ by

$$
\langle\cdot, \cdot\rangle_{\frac{\chi(k)}{r V} H(k)^{\prime}}:=\left\langle\left(I+\epsilon_{k}\right)^{-1} \cdot, \cdot\right\rangle_{H(k)}=\left\langle\cdot,\left(I+\epsilon_{k}\right)^{-1} \cdot\right\rangle_{H(k)},
$$

since $\epsilon_{k}=\epsilon_{k}^{*}$. This implies that $H^{\prime}(k)$ is the Fubini-Study metric on the bundle $\mathcal{E}(k)$ induced from the embedding of $X$ into $G(r, N)$ given by $\left\{S_{\alpha}\right\}$, thus we are in the situation of Section 2. For any $D \in i \mathfrak{s u}(N), \exp D \in$ $S L(N, \mathbb{C})$ will move the projective embedding

$$
X \longrightarrow G(r, N)
$$

given by $\left\{S_{\alpha}\right\}$ to a new position. The Fubini-Study metric on $\mathcal{E}(k)$ induced from this new embedding will be denoted by $H_{D}$. Let $E_{D}$ be the error matrix $\left(\eta_{\alpha \beta}\right)$ for the metric $H_{D}$ considered in Section 3.2 and $H_{\infty}$ be the reference metric.

Proposition 4.1. If $\|D\|_{o p} \leq \frac{1}{10}$ then

i) If

$$
\|D\|_{o p}+\frac{1}{k} \leq R
$$

then the metric $H_{D}$ is $R$-bounded.

ii) There is a constant $C$ such that

$$
\left\|E_{D}\right\|_{o p} \leq \frac{C}{k^{n}}\left(\|D\|_{o p}+\left\|\epsilon_{k}\right\|_{C^{2}}\right) .
$$

Here all norms are with respect to $H_{\infty}^{\prime}$, the Fubini-Study metric induced from $H_{\infty}$.

Proof. The whole construction is $S U(N)$ invariant so we may assume that $D$ is a diagonal matrix $D=\operatorname{diag}\left(\lambda_{\alpha}\right)$. By definition the metric $H_{D}$ is given by $\langle\cdot, \cdot\rangle_{H_{D}}=\left\langle\cdot, B_{D}^{-1} \cdot\right\rangle_{H^{\prime}(k)}$ where

$$
B_{D}:=\sum_{\alpha} S_{\alpha} e^{2 \lambda_{\alpha}} S_{\alpha}^{* H^{\prime}(k)}=\sum_{\alpha} S_{\alpha}\left(e^{2 \lambda_{\alpha}}-1\right) S_{\alpha}^{* H^{\prime}(k)}+I .
$$


The first part of the proposition then follows from

$$
\begin{gathered}
\left\|H^{\prime}(k)-H_{D}\right\|_{C^{r}} \leq C\|D\|_{o p}, \\
\left\|H_{\infty}^{\prime}-H^{\prime}(k)\right\|_{C^{r}} \leq \frac{C}{k} .
\end{gathered}
$$

For the second part, we know that

$$
\begin{aligned}
& \left(S_{\alpha}, S_{\beta}\right)_{H_{D}} \\
= & \left(S_{\alpha}, B_{D}^{-1} S_{\beta}\right)_{H^{\prime}(k)} \\
= & \left(S_{\alpha},\left(B_{D}^{-1}-I\right) S_{\beta}\right)_{H^{\prime}(k)}+\left(S_{\alpha}, S_{\beta}\right)_{H^{\prime}(k)} \\
\leq & C\|D\|_{o p}\left\|S_{\alpha}\right\|_{L^{2}\left(H^{\prime}(k)\right)}\left\|S_{\beta}\right\|_{L^{2}\left(H^{\prime}(k)\right)}+\frac{r V}{\chi(k)}\left(\delta_{\alpha \beta}+\left\|\epsilon_{k}\right\|_{C^{2}}\right) \\
\leq & C\|D\|_{o p}\left\|S_{\alpha}\right\|_{L^{2}\left(H^{\prime}(k)\right)}\left\|S_{\beta}\right\|_{L^{2}\left(H^{\prime}(k)\right)}\left(1+2\left\|\epsilon_{k}\right\|_{C^{2}}\right)+\frac{r V}{\chi(k)}\left(\delta_{\alpha \beta}+\left\|\epsilon_{k}\right\|_{C^{2}}\right) \\
= & C\|D\|_{o p} \frac{r V}{\chi(k)}\left(1+2\left\|\epsilon_{k}\right\|_{C^{2}}\right)+\frac{r V}{\chi(k)}\left(\delta_{\alpha \beta}+\left\|\epsilon_{k}\right\|_{C^{2}}\right)
\end{aligned}
$$

so we get the second part of the proposition.

\subsection{Convergence.}

Now we are ready to state the

Proof of Theorem 1.2. The proof for the first part is straightforward. Suppose that $H_{k} \rightarrow H_{\infty}$ in $C^{\infty}$ then we have for $\forall r>0$

$$
\left\|B_{k}\left(H_{k}\right)-k^{n} \cdot I_{\mathcal{E}}-\left(\frac{\sqrt{-1}}{2 \pi} \bigwedge F_{\left(\mathcal{E}, H_{k}\right)}+\frac{1}{2} S(\omega) \cdot I_{\mathcal{E}}\right) k^{n}\right\|_{C^{r}} \leq C k^{n-2}
$$

for some fixed constant $\mathrm{C}$ (independent of $\mathrm{k}$ ). By hypothesis $H^{(k)}$ is a balanced metric so $B_{k}\left(H_{k}\right)=\frac{\chi(k)}{r V}$ this clearly implies that

$$
\left\|\frac{\sqrt{-1}}{2 \pi} \bigwedge F_{\left(\mathcal{E}, H_{k}\right)}+\frac{1}{2} S(\omega) \cdot I_{\mathcal{E}}-\left(\frac{\operatorname{deg}(\mathcal{E})}{V r}+\frac{1}{2} \bar{s}\right) I_{\mathcal{E}}\right\|_{C^{r}}=O\left(k^{-1}\right),
$$

hence we get the first part.

Conversely, fix any integer $r>0$. First we construct an approximate solution using the Theorem 4.1 such that the error term $E=O\left(k^{-q}\right)$, where $q>0$ is to be determined. Let $k$ be sufficient large then for

$$
\|D\|_{o p}+\left\|\epsilon_{k}\right\|_{C^{2}} \leq \epsilon
$$


with $\epsilon$ determined in Theorem 3.1, Proposition 4.1 implies $H_{D}$ has $R$ bounded geometry and $k^{n}\left\|E_{D}\right\|_{o p} \leq \epsilon$, thus Theorem 3.1 becomes available. By Corollary 3.1, we get $\Lambda_{z} \leq C k^{3+5 n}$ for the corresponding point $z \in Z$. In order to apply the Proposition 2.1 we set $q>3+\frac{11 n}{2}$, then we have

$$
\Lambda_{z}\|E\| \leq \Lambda_{z} \sqrt{\chi(k)}\|E\|_{o p} \leq C k^{3+5 n-q+\frac{n}{2}} \leq \delta .
$$

Note that $\nu(\exp D \cdot z)=E_{D}$, Proposition 2.1 then implies that for large enough $k$, we obtain a solution to our problem with

$$
\|D\|_{o p} \leq\|D\| \leq C k^{3+5 n-q+\frac{n}{2}} \text {. }
$$

Let us denote the induced balanced metric by $H^{(k)}$. Finally we rescale back to $H_{k}:=\frac{\chi(k)}{r V} H^{(k)} \otimes h^{-k}$ giving us

$$
\left\|H_{k}-H_{\infty}\right\|_{C^{r}\left(H_{\infty}\right)} \leq O\left(k^{3+\frac{11 n}{2}+n-q+\frac{r}{2}}\right) .
$$

So if we choose $q>3+\frac{13 n}{2}+\frac{r}{2}$ at the beginning we get the convergence in $C^{r}$.

\section{Appendix: Expansion of Bergman kernel.}

In this section, we compute the the second coefficient in the expansion of the Bergman kernel. The basic idea is due to Tian[T], and later extended by Ruan $[\mathrm{R}]$ and $\mathrm{Lu}[\mathrm{Lu}]$.

\subsection{Statements of the results.}

Let us start with the following theorem proved by Catlin ${ }^{3}$

Theorem 5.1. $[\mathrm{C}]$

1) For fixed Hermitian metric $H$ and $h$ on $\mathcal{E}$ and $\mathcal{O}_{X}(1)$ respectively, there is an asymptotic expansion as $k \rightarrow \infty$

$$
B_{k}(H, h) \sim A_{0}(H, h) k^{n}+A_{1}(H, h) k^{n-1}+\cdots,
$$

where $A_{i}(H, h) \in \Gamma($ EndE) are smooth sections defined locally by $H$.

2)The expansion holds in $C^{\infty}$ in the sense that for any $r, N>0$

$$
\left\|B_{k}(H, h)-\sum_{i=0}^{N} A_{i}(H, h) k^{n-i}\right\|_{C^{r}} \leq K_{r, N, H} k^{n-N-1},
$$

\footnotetext{
${ }^{3}$ Zelditch proved in $[\mathrm{Z}]$ the same statement for line bundle case.
} 
for some constants $K_{r, N, H}$. Moreover the expansion is uniform in that for any $r, N$ there is an integer $r^{\prime}$ such that if $H$ and $h$ runs over a set of metrics which are bounded in $C^{r^{\prime}}$, and with $H$ and $h$ bounded from below, the constants $K_{r, N, H, h}$ are bounded by some $K_{r, N}$ independent of $H$ and $h$.

The main task of this Appendix is to extend Lu's computation to the vector bundle case. More precisely, we have

\section{Theorem 5.2.}

$$
\begin{gathered}
A_{0}(H, h)=I, \\
A_{1}(H, h)=\frac{\sqrt{-1}}{2 \pi} \bigwedge F(\mathcal{E})+\frac{1}{2} S(\omega) \cdot I .
\end{gathered}
$$

where $\bigwedge F(\mathcal{E}) \in \Gamma($ End $\mathcal{E})$ is the contraction of the curvature of $H$ with respect to $\omega:=\frac{\sqrt{-1}}{2 \pi} \operatorname{Ric}(h)$, and $S(\omega)$ is the scalar curvature of $X$ with respect to $\omega$.

\subsection{Constructing Peak sections.}

We start by introducing a canonical coordinate system for both the base manifold and the holomorphic vector bundle over it. The canonical coordinate which was first introduced by Bochner [Bo].

Lemma 5.1. ([R] Proposition 2.1) For any $x \in X$ there is a unique holomorphic coordinate map z up to affine transformation, for which there exist a Kähler potential $K_{x}(z)$ on $X$ such that all the $(0, l),(1, l),(l, 0),(l, 1)$ terms in the Taylor expansion of $K_{x}(z)$ vanish except for $(1,1)$ term which equals to $|z|^{2}$. That is,

$$
K_{x}(z)=|z|^{2}-\frac{1}{4} R_{i \bar{j} k \bar{l}} z_{i} \bar{z}_{j} z_{k} \bar{z}_{l}+O\left(|z|^{2}\right) .
$$

These are the canonical coordinates.

The corresponding bundle version of the above Lemma is the following

Lemma 5.2. Fix any $x \in X$, let us take a neighborhood $U_{x} \ni x$ such that $\left.\mathcal{E}\right|_{U_{x}} \cong U_{x} \times \mathbb{C}^{r}$ is a local trivialization. Then for any holomorphic coordinate system of $U_{x}$, we can find a local holomorphic frame $\left\{S^{i}\right\}_{i=1}^{r}$ of $\mathcal{E}$ over $U_{x}$ such that with respect to this frame, the metric $H$ on $\mathcal{E}$ has the following expansion:

$$
H_{x}=I-H_{i \bar{j}} z_{i} \bar{z}_{j}+O\left(|z|^{3}\right)
$$


where $H_{i \bar{j}} \in \Gamma(\operatorname{Herm}(\mathcal{E}))$ and $H_{i \bar{j}}=\bar{H}_{j \bar{i}}^{t}$. Moreover, for any integer $l>0$ there is a local holomorphic frame $\left\{S^{i}\right\}_{i=1}^{r}$ over $U_{x}$ such that there is no pure $z_{i}$ and $\bar{z}_{i}$ terms of order less than $l$ in the expansion of $H_{x}$.

Proof. We follow the strategy of Bochner. First we choose a holomorphic frame $\left\{S^{i}\right\}_{i=1}^{r}$, then near $x \in X$ we have the following expansion.

$$
\left\langle S^{i}, S^{j}\right\rangle=\delta^{i j}+H_{k}^{i j} z_{k}+H_{\bar{k}}^{i j} \bar{z}_{k}+O\left(|z|^{2}\right) .
$$

Introduce a new basis

$$
\tilde{S}^{i}=S^{i}+a_{k}^{i p} S^{p} z^{k}
$$

We have

$$
\begin{aligned}
\left\langle\tilde{S}^{i}, \tilde{S^{j}}\right\rangle & =\left\langle S^{i}+a_{k}^{i p} S^{p} z_{k}, S^{j}+a_{k}^{j q} S^{q} z_{k}\right\rangle \\
& =\left\langle S^{i}, S^{j}\right\rangle+a_{k}^{i p} z_{k}\left\langle S^{p}, S^{j}\right\rangle+\bar{a}_{k}^{j q} \bar{z}_{k}\left\langle S^{i}, S^{q}\right\rangle+O\left(|z|^{2}\right) \\
& =\delta^{i j}+H_{k}^{i j} z_{k}+H_{\bar{k}}^{i j} \bar{z}_{k}+a_{k}^{i p} z_{k}\left(\delta^{p j}+H_{m}^{p j} z_{m}+H_{\bar{m}}^{p j} \bar{z}_{m}\right) \\
& +\bar{a}_{k}^{j q} \bar{z}_{k}\left(\delta^{i q}+H_{m}^{i q} z_{m}+H_{\bar{m}}^{i q} \bar{z}_{m}\right)+O\left(|z|^{2}\right) \\
& =\delta^{i j}+\left(H_{k}^{i j}+a_{k}^{i j}\right) z_{k}+\left(H_{\bar{k}}^{i j}+\bar{a}_{k}^{j i}\right) \bar{z}_{k}+O\left(|z|^{2}\right),
\end{aligned}
$$

since we have $\left\langle S^{i}, S^{j}\right\rangle=\overline{\left\langle S^{j}, S^{i}\right\rangle}$ which implies $\bar{H}_{k}^{j i}=H_{\bar{k}}^{i j}$. So if we set $a_{k}^{i j}=-H_{k}^{i j}$, we will have $\left\langle\tilde{S}^{i}, \tilde{S}^{j}\right\rangle=\delta^{i j}+O\left(|z|^{2}\right)$. We killed the first order terms. For the second order terms, suppose we have

$$
\left\langle S^{i}, S^{j}\right\rangle=\delta^{i j}+H_{k l}^{i j} z_{k} z_{l}+H_{\bar{k} l}^{i j} \bar{z}_{k} \bar{z}_{l}-H_{k \bar{l}}^{i j} z_{k} \bar{z}_{l}-H_{\bar{k} l}^{i j} \bar{z}_{k} z_{l}+O\left(|z|^{3}\right)
$$

Introduce a new basis

$$
\tilde{S}^{i}=S^{i}+a_{k l}^{i p} S^{p} z_{k} z_{l}
$$

we have

$$
\begin{aligned}
\left\langle\tilde{S}^{i}, \tilde{S^{j}}\right\rangle & =\left\langle S^{i}+a_{k l}^{i p} S^{p} z_{k} z_{l}, S^{j}+a_{k l}^{j q} S^{q} z_{k} z_{l}\right\rangle \\
& =\left\langle S^{i}, S^{j}\right\rangle+a_{k l}^{i p} z_{k} z_{l}\left\langle S^{p}, S^{j}\right\rangle+\bar{a}_{k l}^{j q} \bar{z}_{k} \bar{z}_{l}\left\langle S^{i}, S^{q}\right\rangle+O\left(|z|^{3}\right) \\
& =\delta^{i j}+H_{k l}^{i j} z_{k} z_{l}+H_{\overline{k l}}^{i j} \bar{z}_{k} \bar{z}_{l}+a_{k l}^{i p} z_{k} z_{l}\left(\delta^{p j}+O\left(|z|^{2}\right)\right) \\
& +\bar{a}_{k l}^{j q} \bar{z}_{k} \bar{z}_{l}\left(\delta^{i q}+O\left(|z|^{2}\right)\right)+O\left(|z|^{3}\right) \\
& =\delta^{i j}+\left(H_{k l}^{i j}+a_{k l}^{i j}\right) z_{k} z_{l}+\left(H_{\bar{k} l}^{i j}+\bar{a}_{k l}^{j i}\right) \bar{z}_{k} \bar{z}_{l}+O\left(|z|^{3}\right) .
\end{aligned}
$$


Again $\left\langle S^{i}, S^{j}\right\rangle=\overline{\left\langle S^{j}, S^{i}\right\rangle}$ implies $\bar{H}_{k l}^{j i}=H_{\bar{k} \bar{l}}^{i j}$, so if we set $a_{k l}^{i j}=-H_{k l}^{i j}$ we will be able to kill the second order terms. By continuing this process we will be able to get rid of the pure $z_{i}$ and $\bar{z}_{i}$ terms up to any order.

Remark 5.1. The idea of using holomorphic gauge to kill the holomorphic and anti-holomophic terms is essentially the gauge fixing.

Corollary 5.1. Under the canonical coordinate and canonical framing, we have the curvature form

$$
F(\mathcal{E})_{x}=H_{i j} d z_{i} \wedge d \bar{z}_{j}
$$

Proof.

$$
\begin{aligned}
F & =\bar{\partial}\left(H^{-1} \partial H\right) \\
& =\bar{\partial}\left(\left(I+H_{i \bar{j}} z_{i} \bar{z}_{j}+O\left(|z|^{3}\right)\right) \partial\left(I-H_{i \bar{j}} z_{i} \bar{z}_{j}+O\left(|z|^{3}\right)\right)\right) \\
& =-\bar{\partial}\left(H_{i \bar{j}} \bar{z}_{j} d z_{i}+O\left(|z|^{2}\right)\right) \\
& =-H_{i \bar{j}} d \bar{z}_{j} d z_{i}+O\left(|z|^{2}\right) \\
& =H_{i \bar{j}} d z_{i} d \bar{z}_{j}+O\left(|z|^{2}\right) .
\end{aligned}
$$

To be able to evaluate Bergman kernel at a given point, we need following two propositions.

Proposition 5.1. [T] Fix a point $x \in X$, let $\left\{S^{i}\right\}_{i=1}^{r}$ be a canonical frame for $\mathcal{E}$ and $e$ a canonical section for $\mathcal{O}_{X}(1)$ in a neighborhood of $x$. Let the $z_{i}$ 's give a local canonical co-ordinates for $\mathrm{x}$.

Then for $P=\left(p_{1}, \cdots, p_{n}\right) \in \mathbb{Z}_{+}^{n}$, and a integer $p^{\prime}>p_{1}+\cdots+p_{n}$, there exists an $k_{0}>0$ such that for $k>k_{0}$, there are holomorphic sections $S_{P, k}^{p^{\prime}, i} \in H^{0}(X, \mathcal{E}(k))$ for $1 \leq i \leq r$ satisfying:

$$
\begin{gathered}
\int_{X}\left\|S_{P, k}^{p^{\prime}, i}\right\|_{H \otimes h^{k}}^{2} d V=1 \\
\int_{X-\left\{|z| \leq \frac{\log k}{\sqrt{k}}\right\}}\left\|S_{P, k}^{p^{\prime}, i}\right\|_{H \otimes h^{k}}^{2} d V \leq O\left(\frac{1}{k^{2 p^{\prime}}}\right),
\end{gathered}
$$

and locally at $x \in X$

$$
S(z)_{P, k}^{p^{\prime}, i}=\lambda_{\left(p_{1}, \cdots, p_{n}\right)}\left\{z_{1}^{p_{1}} \cdots z_{n}^{p_{n}}+O\left(|z|^{2 p^{\prime}}\right)\right\} e^{k} \otimes S^{1}\left(1+O\left(\frac{1}{k^{2 p^{\prime}}}\right)\right) .
$$


Now let $P$ stand for the multi-index $\left(p_{1}, \ldots, p_{n}\right)$, and set $z^{P}:=z_{1}^{p_{1}} \cdots z_{n}^{p_{n}}$, similarly $z^{Q}:=z_{1}^{q_{1}} \cdots z_{n}^{q_{n}}$, then we have

Proposition 5.2. $[\mathrm{R}]$ We continue with the notations and assumptions of Proposition 5.1. Let $T$ be another section of $\mathcal{E}(k)$. Locally around 0 we have

$$
\begin{gathered}
T=e^{k} \otimes \sum_{i=1}^{r} f_{i} S^{i} \\
S_{P, k}^{p^{\prime}}(z)=\lambda_{\left(p_{1}, \cdots, p_{n}\right)}\left\{z_{1}^{p_{1}} \cdots z_{n}^{p_{n}}+O\left(|z|^{2 p^{\prime}}\right)\right\} e^{k} \otimes S^{1}\left(1+O\left(\frac{1}{k^{2 p^{\prime}}}\right)\right)
\end{gathered}
$$

i) If $f_{1}(z)$ 's Taylor expansion around 0 doesn't have the monomial $z_{1}^{p_{1}} \cdots z_{n}^{p_{n}}$, then

$$
\left(S_{P, k}^{p^{\prime}}, T\right)_{h^{k} \otimes H}=O\left(\frac{1}{k}\right)\left\|S_{P, k}^{p^{\prime}}\right\|_{h^{k} \otimes H}\|T\|_{h^{k} \otimes H} .
$$

ii)If $f_{1}(z)$ 's Taylor expansion around 0 doesn't have terms $z^{Q}$ with $q<p+d$ where $q=q_{1}+\cdots+q_{n}, p=p_{1}+\cdots+p_{n},(d \geq 1)$, and $f_{2}(0)=\cdots=f_{r}(0)=0$ then

$$
\left(S_{P, k}^{p^{\prime}}, T\right)_{h^{k} \otimes H}=O\left(\frac{1}{k^{1+\frac{d}{2}}}\right)\left\|S_{P, k}^{p^{\prime}}\right\|_{h^{k} \otimes H}\|T\|_{h^{k} \otimes H}
$$

Proof. The proof follows directly from Lemma 3.2 in $[\mathrm{R}]$.

\subsection{Evaluating $A_{1}(H, h)$.}

With the preparation in the previous section understood, we are ready to calculate the first two coefficients of $B$ as in [Lu]. Let $N:=\operatorname{dim} H^{0}(X, \mathcal{E}(k))$ and $\left\{S_{1}, \cdots, S_{N}\right\}$ be a basis for $H^{0}(X, \mathcal{E}(k))$. Let us introduce the $L^{2}$-metric on $H^{0}(X, \mathcal{E}(k))$ as the following

$$
\left(S_{A}, S_{B}\right)_{h^{k} \otimes H}:=\int_{X}\left\langle S_{A}, S_{B}\right\rangle d V
$$

Now let us fix the notation, $1 \leq A, B, C, D, E \leq N$ and $1 \leq i, j, l \leq r$, where $r$ is the rank of $\mathcal{E}$. Let us define

$$
\Pi_{A B}:=\left(S_{A}, S_{B}\right)_{h^{k} \otimes H}
$$


then there exists a matrix $\Theta$ such that $\Pi=\Theta \Theta^{*}$. Let $\Xi:=\Theta^{-1}$ and set $T_{A}=\sum_{B} \Xi_{A B} S_{B}$, then we have

$$
\begin{aligned}
\left(T_{A}, T_{B}\right)_{h^{k} \otimes H} & =\left(\sum_{C} \Xi_{A C} S_{C}, \sum_{D} \Xi_{B D} S_{D}\right)_{h^{k} \otimes H} \\
& =\sum_{C, D} \Xi_{A C} \bar{\Xi}_{B D}\left(S_{C}, S_{D}\right)_{h^{k} \otimes H} \\
& =\sum_{C, D} \Xi_{A C} \bar{\Xi}_{B D} \Pi_{C D} \\
& =\sum_{C, D, E} \Xi_{A C} \bar{\Xi}_{B D} \Theta_{C E} \bar{\Theta}_{D E} \\
& =\delta_{A B},
\end{aligned}
$$

that is $\left\{T_{A}\right\}_{A=1}^{N}$ is an orthonormal basis for $H^{0}(X, \mathcal{E}(k))$. The Bergman kernel $B:=\sum_{A} T_{A} T_{A}^{*}$. Fix a point $x \in X$, let $\left\{e_{i}\right\}_{i=1}^{r}$ be a local orthonormal frame in a neighborhood of $x$. Locally we have $S_{A}=\sum_{i} S_{A i} e_{i}$, so $T_{A}=$ $\sum_{i} T_{A i} e_{i}=\sum_{B, i} \Xi_{A B} S_{B i} e_{i}$. Since

$$
B e_{i}=\sum_{l} B_{i l} e_{l}=\sum_{A=1}^{N} T_{A}\left\langle e_{i}, T_{A}\right\rangle=\sum_{A, l} T_{A l} \bar{T}_{A i} e_{l}
$$

we have $B_{i l}=\sum_{A=1}^{N} \bar{T}_{A i} T_{A l}$. Put this into the matrix form we have the following

$$
\begin{aligned}
& B_{x}=\left(\begin{array}{ccc}
B_{11} & \ldots & B_{1 r} \\
\ldots \ldots & \ldots & \ldots \\
B_{r 1} & \ldots & B_{r r}
\end{array}\right) \\
& =\left(\begin{array}{ccc}
\bar{T}_{11} & \ldots & \bar{T}_{N 1} \\
\ldots \ldots & \ldots & \ldots \\
\bar{T}_{1 r} & \ldots & \bar{T}_{N r}
\end{array}\right)\left(\begin{array}{ccc}
T_{11} & \ldots & T_{1 r} \\
\ldots \ldots & \ldots & \ldots \\
T_{N 1} & \ldots & T_{N r}
\end{array}\right) \\
& =\left(\begin{array}{ccc}
\bar{S}_{11} & \ldots & \bar{S}_{N 1} \\
\ldots \ldots & \ldots & \ldots \\
\bar{S}_{1 r} & \ldots & \bar{S}_{N r}
\end{array}\right)\left(\begin{array}{ccc}
\bar{\Xi}_{11} & \ldots & \bar{\Xi}_{N 1} \\
\ldots \ldots & \ldots & \ldots \\
\bar{\Xi}_{1 N} & \ldots & \bar{\Xi}_{N N}
\end{array}\right) \\
& \cdot\left(\begin{array}{ccc}
\Xi_{11} & \ldots & \Xi_{1 N} \\
\ldots \ldots & \ldots & \ldots \\
\Xi_{N 1} & \ldots & \Xi_{N N}
\end{array}\right)\left(\begin{array}{ccc}
S_{11} & \ldots & S_{1 r} \\
\ldots & \ldots & \ldots \\
S_{N 1} & \ldots & S_{N r}
\end{array}\right)
\end{aligned}
$$


Note that if we choose the basis $S_{1}, \cdots, S_{N}$ such that at $x$,

$$
\left(\begin{array}{ccc}
S_{11} & \ldots & S_{N 1} \\
\ldots \ldots & \ldots & \ldots \\
S_{1 r} & \ldots & S_{N r}
\end{array}\right)=\left(\begin{array}{cccccc}
1 & \ldots & 0 & 0 & \ldots & 0 \\
\vdots & \ddots & \vdots & \vdots & \ddots & \vdots \\
0 & \ldots & 1 & 0 & \ldots & 0
\end{array}\right)
$$

then we have

$$
B_{x}=\left(\begin{array}{ccc}
\Xi_{11} & \ldots & \bar{\Xi}_{N 1} \\
\ldots \ldots & \ldots & \ldots \\
\bar{\Xi}_{1 r} & \ldots & \bar{\Xi}_{N r}
\end{array}\right)\left(\begin{array}{ccc}
\Xi_{11} & \ldots & \Xi_{1 r} \\
\ldots \ldots & \ldots & \ldots \\
\Xi_{N 1} & \ldots & \Xi_{N r}
\end{array}\right)
$$

Since $\Pi=\Theta \Theta^{*}, \Pi^{-1}=\left(\Theta^{*}\right)^{-1}(\Theta)^{-1}=\Xi^{*} \Xi$, we have

$$
B_{x}=\left(\begin{array}{ccc}
\Lambda_{11} & \ldots & \Lambda_{1 r} \\
\ldots \ldots & \ldots & \ldots \\
\Lambda_{r 1} & \ldots & \Lambda_{r r}
\end{array}\right)
$$

where $\Lambda=\Pi^{-1}$. So our task is to estimate $\Lambda$ at the point $x$.

Proof of Theorem 5.2. For each fixed point $x \in X$, take $U_{x}$ to be a neighborhood of $x$ such that $\left.\mathcal{E}\right|_{U_{x}}$ is trivial. Choose a canonical co-ordinate $\left\{z_{i}\right\}$ 's for $U_{x}$, and a canonical frame of $\mathcal{E}$ over $U_{x}$. Now we can apply Proposition 5.1 to get sections $\left\{S_{0, k}^{p^{\prime}, i}\right\}_{i=1}^{r} \in H^{0}(\mathcal{E}(k))$. Extend these to a basis

$$
\left\{S_{0, k}^{p^{\prime}, 1}, \cdots, S_{0, k}^{p^{\prime}, r}, S_{r+1}, \cdots, S_{N}\right\} \in H^{0}(\mathcal{E}(k))
$$

in such a way that $S_{r+1}(x)=\cdots=S_{N}(x)=0$. By our choice, we have that $\left\{S_{0, k}^{p^{\prime}, i}\right\}_{i=1}^{r}$ is an orthonormal basis for $\mathcal{E}_{x}$. So to evaluate $B_{x}$ we only need to evaluate the upper-left $r \times r$ part of the matrix $\Pi$, which is the following 


$$
\begin{aligned}
& \int_{X}\left(\begin{array}{llr}
\left\langle S_{0, k}^{p^{\prime}, 1}, S_{0, k}^{p^{\prime}, 1}\right\rangle & \ldots & \left\langle S_{0, k}^{p^{\prime}, 1}, S_{0, k}^{p^{\prime}, r}\right\rangle \\
\ldots \ldots \ldots \ldots \ldots \ldots & \ldots \ldots \ldots \ldots \\
\left\langle S_{0, k}^{p^{\prime}, r}, S_{0, k}^{p^{\prime}, 1}\right\rangle & \ldots & \left\langle S_{0, k}^{p^{\prime}, r}, S_{0, k}^{p^{\prime}, r}\right\rangle
\end{array}\right)_{r \times r} d V+O\left(e^{-(\log k)^{2}}\right) \\
& =\int_{|z|^{2} \leq \frac{(\log k)^{2}}{k}}\left(\begin{array}{lll}
\left\langle S_{0, k}^{p^{\prime}, 1}, S_{0, k}^{p^{\prime}, 1}\right\rangle & \ldots & \left\langle S_{0, k}^{p^{\prime}, 1}, S_{0, k}^{p^{\prime}, r}\right\rangle \\
\ldots \ldots \ldots \ldots \ldots \ldots & \ldots \ldots \ldots \ldots \\
\left\langle S_{0, k}^{p^{\prime}, r}, S_{0, k}^{p^{\prime}, 1}\right\rangle & \ldots & \left\langle S_{0, k}^{p^{\prime}, r}, S_{0, k}^{p^{\prime}, r}\right\rangle
\end{array}\right) d V+O\left(e^{-(\log k)^{2}}\right) \\
& =\int_{|z|^{2} \leq \frac{(\log k)^{2}}{k}} h^{k} \cdot H d V+O\left(e^{-(\log k)^{2}}\right) \\
& =\int_{|z|^{2} \leq \frac{(\log k)^{2}}{k}} e^{-k\left(|z|^{2}-\frac{1}{4} R_{i \bar{j} k \bar{l}} z_{i} \bar{z}_{j} z_{k} \bar{z}_{l}+O\left(|z|^{5}\right)\right)}\left(I-H_{i \bar{j}} z_{i} \bar{z}_{j}+O\left(|z|^{3}\right)\right) d V \\
& +O\left(e^{-(\log k)^{2}}\right) \\
& =\int_{|z|^{2} \leq \frac{(\log k)^{2}}{k}} e^{-k|z|^{2}}\left(1-\frac{k}{4} R_{i \bar{j} k \bar{l}} z_{i} \bar{z}_{j} z_{k} \bar{z}_{l}+k O\left(|z|^{5}\right)\right)\left(I-H_{i \bar{j}} z_{i} \bar{z}_{j}\right. \\
& \left.+O\left(|z|^{3}\right)\right) d V+O\left(e^{-(\log k)^{2}}\right) \\
& =\int_{|z|^{2} \leq \frac{(\log k)^{2}}{k}} e^{-k|z|^{2}}\left(1-H_{i \bar{j}} z_{i} \bar{z}_{j}+\frac{k}{4} R_{i \bar{j} k \bar{l}} z_{i} \bar{z}_{j} z_{k} \bar{z}_{l}\right. \\
& \left.+O\left(|z|^{3}\right)+k O\left(|z|^{5}\right)\right) e^{\log \operatorname{det} g} d V_{0}+O\left(e^{-(\log k)^{2}}\right) \\
& =\int_{|z|^{2} \leq \frac{(\log k)^{2}}{k}} e^{-k|z|^{2}-R_{i \bar{j}} z_{i} \bar{z}_{j}+O\left(|z|^{3}\right)}\left(1-H_{i \bar{j}} z_{i} \bar{z}_{j}+\frac{k}{4} R_{i \bar{j} k l} z_{i} \bar{z}_{j} z_{k} \bar{z}_{l}\right. \\
& \left.+O\left(|z|^{3}\right)+k O\left(|z|^{5}\right)\right) d V_{0}+O\left(e^{-(\log k)^{2}}\right) \\
& =\int_{|z|^{2} \leq \frac{(\log k)^{2}}{k}} e^{-k|z|^{2}+O\left(|z|^{3}\right)}\left(1-H_{i \bar{j}} z_{i} \bar{z}_{j}+\frac{k}{4} R_{i \bar{j} k l} z_{i} \bar{z}_{j} z_{k} \bar{z}_{l}-R_{i \bar{j}} z_{i} \bar{z}_{j}\right. \\
& \left.+O\left(|z|^{3}\right)+k O\left(|z|^{5}\right)\right) d V_{0}+O\left(e^{-(\log k)^{2}}\right) \\
& =\frac{1}{k^{n}}-\frac{1}{k^{n+1}} \sum_{i} H_{i \bar{i}}+\frac{k}{2} \cdot \frac{S(\omega)}{k^{n+2}}-\frac{S(\omega)}{k^{n+1}}+O\left(\frac{1}{k^{n+2}}\right) \\
& =\frac{1}{k^{n}}-\frac{1}{k^{n+1}}\left(\frac{\sqrt{-1}}{2 \pi} \bigwedge F(\mathcal{E})+\frac{1}{2} S(\omega) \cdot I\right)+O\left(\frac{1}{k^{n+2}}\right) \text {. }
\end{aligned}
$$

To get the eighth identity we have used the fact that if $i \neq j$ then the integral of $H_{i \bar{j}} z_{i} \bar{z}_{j}$ vanishes and the formula

$$
\int_{\mathbb{C}^{n}}\left|z_{1}^{p_{1}} \cdots z_{n}^{p_{n}}\right|^{2} e^{-k|z|^{2}} d V_{0}=\frac{p_{1} ! \cdots p_{n} !}{k^{n+p}}
$$


with $d V_{0}:=\left(\frac{\sqrt{-1}}{2 \pi}\right)^{n} d z_{1} \wedge d \bar{z}_{1} \wedge \cdots \wedge d z_{n} \wedge d \bar{z}_{n}$. Also a priori there will be

terms of order $O\left(\frac{1}{K^{n+\frac{3}{2}}}\right)$ coming from the integral of $O\left(|z|^{3}\right)+k O\left(|z|^{5}\right)$. They vanish because the number of $z$ 's does not match the number of $\bar{z}$ 's for terms with odd degree (This was also observed in $[\mathrm{Lu}]$ ). The last identity follows from Corollary 5.1.

To get $B_{x}$, we only need to invert $\Pi$. Our conclusion then follows from Proposition (5.2 ii) and the fact that for any traceless matrix $A$

$$
\operatorname{det}(I+t A)=1+O\left(t^{2}\right)
$$

\section{References.}

[AB] M. Atiyah and R. Bott, The Yang-Mills equations over Riemann surfaces, Philos. Trans. Roy. Soc. London Ser. A, 308(1982), 524-615.

[Bo] S. Bochner, Curvature in Hermitian metric, Bull. Amer. Math. Soc., 53(1947), 179-195

[C] D.W. Catlin, The Bergman kernel and a theorem of Tian, Analysis and geometry in several complex variables (Katata, 1997), 1-23, Trends Math., Birkhäuser Boston, Boston, MA, 1999.

[D1] - Geometry in Oxford c. 1980-85. Sir Michael Atiyah: a great mathematician of the twentieth century. Asian J. Math.3(1999),no.1 xliii-xlvii.

[D2] - Scalar curvature and projective embeddings,I. J. Diff. Geom. 59(2001), no. 3, 479-522.

[K] S. Kobayashi, Differential Geometry of Complex Vector Bundles Princeton University Press, 1987.

[Lu] Z. Lu, On the lower order terms of the asymptotic expansion of TianYau-Zelditch, Amer. J. Math. 122(2000), no. 2, 235-273.

[OSS] C. Okonek, M. Schneider, and H. Spindler, Vector Bundles on Complex Projective Spaces, Birkhäser, 1980.

[R] W. Ruan, Canonical coordinates and Bergmann metrics, Comm. Anal. Geom. 6 (1998), no.3, 589-631. 
[T] G. Tian On a set of polarized Kahler metrics on algebraic manifolds, J. Diff. Geom. 32(1990), no. 1, 99-130.

[UY] K. Uhlenbeck and S.-T. Yau On the existence of Hermitian YangMills connections on stable vector bundles, Commun. Pure Appl. Math. 39(1986), 257-293.

[W] - Balance point and stability of vector bundles over a projective manifold, Math. Res. Lett. 9(2002), no.2-3, 393-411.

[W1] X. Wang Canonical metric and stability of vector bundles over a projective manifold, Ph.D. Thesis, Brandeis University, 2002.

[Z] S. Zelditch, Szego kernels and a theorem of Tian, Internat. Math. Res. Notices, no. 6, (1998), 317-331.

Department of Mathematics, UCLA

Los ANGELEs,CA 90095-1555

xiaowei@math. ucla.edu

Received February 14, 2004. 